Clemson University

TigerPrints

Publications

Bioengineering

$10-2017$

Multiscale Modeling of Cardiovascular Flows

Alison Marsden

Ethan Kung

Follow this and additional works at: https://tigerprints.clemson.edu/bioengineering_pubs

Part of the Biomedical Engineering and Bioengineering Commons 


\title{
Multiscale Modeling of Cardiovascular Flows
}

\author{
Alison Marsden and Ethan Kung
}

\section{Citation:}

Marsden A, Kung E, "Multi-scale Modeling of Cardiovascular Flows"

Computational Bioengineering, CRC Press Taylor \& Francis Group, Editor: Guigen Zhang 


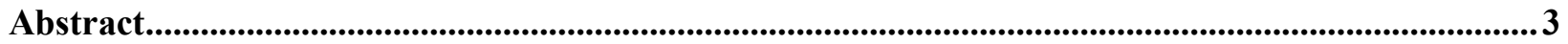

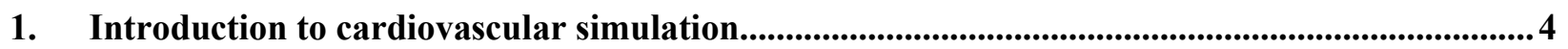

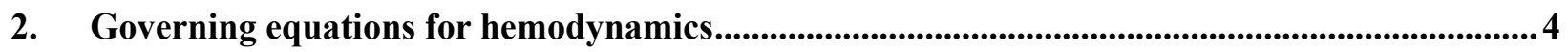

3. Current methods for patient specific modeling ........................................................................................5

3.1 Patient Specific Model Construction ..............................................................................................5 5

3.2 Computational methods for blood flow simulation ..................................................................... 5

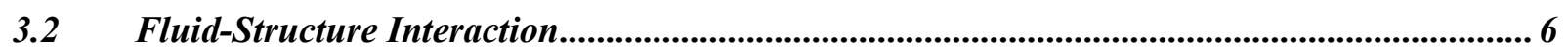

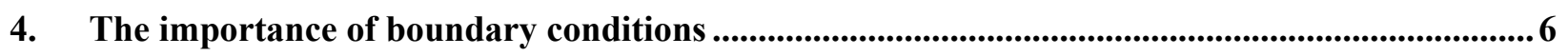

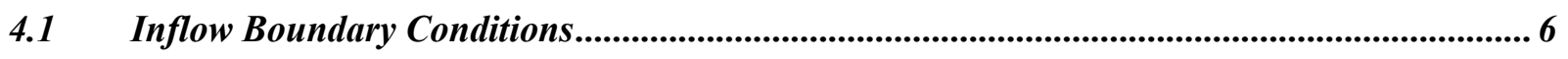

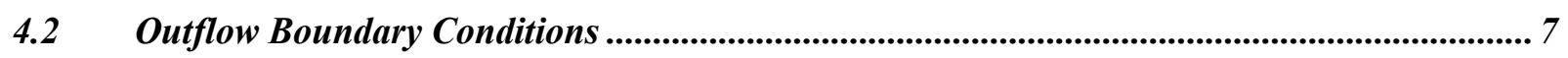

5. Modeling the circulation with a lumped parameter network ............................................................8

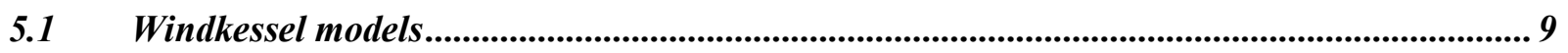

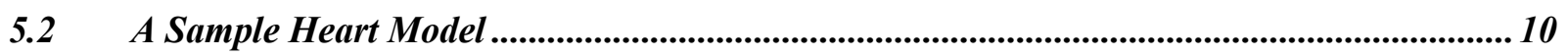

5.3 A Coronary Artery Lumped Model ....................................................................................................... 11

6. Modeling the circulation with the 1-D equations of Blood Flow...............................................12

7. Closed-loop Multi-scale Simulations ….................................................................................................... 12

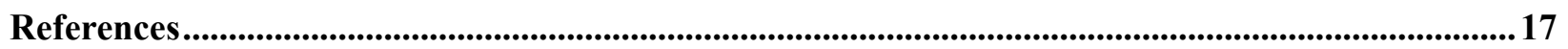




\begin{abstract}
Simulations of blood flow in the cardiovascular system offer investigative and predictive capabilities to augment current clinical tools. Using image-based modeling, the Navier-Stokes equations can be solved to obtain detailed 3-dimensional hemodynamics in patient-specific anatomical models. Relevant parameters such as wall shear stress and particle residence times can then be calculated from the 3D results and correlated with clinical data for treatment planning and device evaluation. Reduced-order models such as open or closed loop 0D lumpedparameter models can simulate the dynamic behavior of the circulatory system using an analogy to electrical circuits. When coupled to 3D simulations as boundary conditions, they produce physiologically realistic pressure and flow conditions in the 3D domain. We describe fundamentals and current state of the art of patient-specific, multi-scale computational modeling approaches applied to cardiovascular disease. These tools enable investigations of hemodynamics reflecting individual patients physiology, and we provide several illustrative case studies. These methods can supplement current clinical measurement and imaging capabilities and provide predictions of patient outcomes for surgical planning and risk stratification.
\end{abstract}




\section{Introduction to cardiovascular simulation}

Simulations of blood flow in the cardiovascular system offer a means to augment current medical imaging modalities and physician experience to potentially improve treatment outcomes for a range of cardiovascular diseases. Perhaps more importantly, simulations offer predictive capabilities to test new surgical concepts, medical devices, and post-operative surgical outcomes. Recent advances in computing technology and efficient algorithms have led to increasingly realistic and accurate simulations, which now capture physiologic levels of blood pressure, detailed anatomy, feedback mechanisms of the circulatory system, and vessel wall deformations.[1-4]

Early work in fluid mechanics of blood flow produced analytical solutions of the NavierStokes equations for pulsatile flow in rigid and elastic tubes. These solutions were derived by J.R. Womersley in the 1950s and are now widely used, in particular as boundary conditions for large-scale flow simulations.[5-10] Following this, starting in the 1960s, lumped parameter models of the circulatory system were developed by analogy to electrical circuits.[11-13] These models have the advantage that they are governed by ordinary differential equations and can be readily solved in near real time on a desktop computer. While they do not provide spatial information, they can be quite complex, providing a surprisingly realistic representation of circulatory dynamics. The one-dimensional equations of blood flow (introduced by Hughes and others in the 1970s) also offer an attractive means to obtain near real-time solutions of circulatory flow dynamics, and can be coupled to 3D solvers as boundary conditions.[14, 15]

The advent of patient specific modeling in the 1990s paved the way for increasingly detailed flow and pressure information to be solved on an individualized basis. Starting from patient image data (typically MRI or CT) a three dimensional model is constructed to represent a portion of the anatomy, often including a diseased region of interest.[16-20] Since only a portion of a patient's anatomy can be included in the 3D model, both due to computational expense and limits of image resolution, boundary conditions must be applied at inlets and outlets of the model to accurately represent the vascular network outside. Patient specific modeling has been applied to a wide range of cardiovascular disease applications, including abdominal aortic aneurysms, cerebral aneurysms, coronary artery disease, heart valves, and congenital heart disease.[3, 4, 21, 22] We first outline the procedures for patient specific modeling and available methods for flow simulations. We then outline the basic mathematical formulations for boundary conditions and multiscale modeling methods used in computational fluid dynamics simulations of blood flow.

\section{Governing equations for hemodynamics}

Blood flow is governed by the Naiver-Stokes equations, which are, for an incompressible, Newtonian fluid,

$$
\nabla u=0 .
$$

While non-Newtonian effects are known to be important in specific scenarios, for example in capillary flow, we focus here on Newtonian flows, which is a widely accepted assumption for 
large vessel hemodynamics.[23]

These equations can be solved numerically using finite volume or finite element methods, among others, with appropriate boundary and initial conditions. This typically requires high performance parallel computing, with significant computational requirements. Issues that must be addressed when choosing a numerical method for the CFD solver include mesh resolution, complex geometry, use of artificial dissipation, and ability to resolve small-scale structures in the flow.

\section{Current methods for patient specific modeling}

\subsection{Patient Specific Model Construction}

Patient specific simulations are typically performed on three-dimensional models of vascular anatomy that are derived from patient image data. Depending on the disease application, either CT or MRI data is typically used. Image data may be segmented using 2D or 3D level set or thresholding methods.[24-26] Common packages for image segmentation include open source packages such as Simvascular (simtk.org) or ITK-SNAP, or commercial packages such as Mimics (Materialise, Leuven, Belgium). The typical steps for model construction using 2D methods are 1) create paths along vessels of interest, 2) manually or automatically draw segmentations of the vessel lumen at discrete locations along the paths, 3) loft (interpolate) the segmentations together to create a 3D solid model, and 4) mesh the model for use with a CFD solver. The typical steps for model construction using a 2D method are shown in Figure 1 for a patient specific model of the normal cerebral vasculature. Advantages of 2D methods are the ability to smoothly represent vascular tree-like branching patterns, such as occur in the pulmonary arteries. Advantages of 3D methods include the ability to more readily capture details of local complex geometry, particularly in aneurysms or complex surgical connections. Ultimately, it is likely a combination of both methods that will prove most advantageous in future work. While automated methods do exist for both 2D and 3D segmentation, noise in the image data often leads to significant user intervention and the need for manual segmentation. Improvements in automated segmentation methods, for example by applying machine learning algorithms, would greatly improve efficiency, reduce user input, and enable high-throughput model construction for clinical applications.[27]

\subsection{Computational methods for blood flow simulation}

Both finite element and finite volume methods have been used to solve the Navier-Stokes equations in cardiovascular applications. Finite element methods (FEM), which are most widely used, are well suited to complex geometries with unstructured meshes. Most recent work has used stabilized (SUPG) methods with linear elements, but higher order elements have also been employed.[28-30] In the FEM approach, the weak form of the Navier-Stokes equations is solved. While commercial solvers can be used on a limited basis, custom solvers offer more flexibility and control over boundary condition implementation, which is essential for obtaining an accurate solution.

Immersed boundary methods, introduced by Peskin in the 1970s, offer an attractive alternative in many situations, and have been successfully applied to heart mechanics and device design.[31-33] These methods offer the advantage of using structured grids for improved solver 
efficiency, but the disadvantage of limited mesh resolution in key areas such as near-wall boundary layers. They are particularly attractive for fluid-structure interaction problems. Unstructured finite volume methods also offer an attractive alternative to FEM methods, particularly with non-commercial codes that do not require additional stabilization terms. These methods have been shown to more accurately capture cycle to cycle variations in unsteady flow in aneurysms, showing surprising evidence of turbulence at low Reynolds numbers.[34, 35]

\subsection{Fluid-Structure Interaction}

Deformation of the vessel walls can be included via fluid-structure interaction (FSI) simulations, in which the solid and fluid domain problems are solved simultaneously using either iterative coupling or strong coupling, as in arbitrary lagrangian-eulerian (ALE) methods. Strongly coupled methods are particularly attractive for large deformation problems, including membrane buckling, valves and ventricular mechanics.[36, 37] However, their computational cost can make them prohibitively expensive for some applications. Immersed boundary methods offer an attractive alternative, which allows for use of structured mesh solvers in problems with complex geometries. Other approaches to reduce computational cost include the coupled momentum method of Figueroa and Taylor, which relies on a small deformation approximation based on Womersley theory to impose forces on the fluid domain without requiring mesh motion.[1, 38]

\section{The importance of boundary conditions}

The choice of boundary conditions is of paramount importance in cardiovascular simulations, as the local flow dynamics are greatly influenced by conditions upstream and downstream of the 3D model. Numerous studies have demonstrated drastic differences in flow solutions, even with simple geometries, and particularly in models with multiple outlets.[2, 39, 40] Commonly used outlet boundary conditions such as zero-pressure or zero-traction, while easiest to implement, are well known to lead to unrealistic solutions, in part because of their inability to capture physiologic levels of pressure. These methods should not be used for fluid-structure interaction problems where the wall deformation depends directly on the pressure level in the vessel.

\subsection{Inflow Boundary Conditions}

A typical model with inlets and outlets requiring a variety of boundary conditions is shown in Figure 2. Inflow boundary conditions are typically enforced at the inlet of the 3D model, and three choices are commonly available. The simplest choice is to use a plug flow condition, applying a uniform velocity profile. However, this assumption is known to be invalid for flow in a pipe, particularly in unsteady flow. A more realistic choice is to impose a parabolic flow profile using the Poiseuille solution for flow in a circular pipe, a well known analytical solution of the Navier-Stokes equations in cylindrical coordinates where the axial component of velocity $u_{z}$ is

Further improvement can be made by using the analytical solution for pulsatile flow in a pipe of Womersley, which is given by 


$$
u_{z}(r, t)=\frac{A}{i \rho \omega}\left[1-\frac{J_{0}\left(i^{\frac{3}{2}} r \sqrt{\frac{\omega}{v}}\right)}{J_{0}\left(R \sqrt{\frac{\omega}{v} i^{\frac{3}{2}}}\right)}\right] e^{i \omega t}
$$

where $A$ is the amplitude of sinusoidal pressure oscillation, $\omega$ is the frequency, $I_{0}$ is the tabulated

Bessel function of the first kind, and $\mathrm{R}$ is the vessel radius. Full derivation of this solution as well as the solution for a general waveform and for an elastic tube has been presented by Zamir and others.[5, 41, 42]

\subsection{Outflow Boundary Conditions}

The choice of outflow boundary conditions in cardiovascular modeling is a much more complex issue compared to the choice of inflow conditions. Typical choices include zero pressure or zero traction conditions applied at the outlet, resistance or impedance conditions, reduced order models which can be open or closed loop, or reduced order one-dimensional wave propagation equations. It is desirable to choose a boundary condition that can capture physiologic levels of pressure, particularly for applications involving deformable walls. In this section, we outline several common choices for outflow boundary condition prescription using zero-pressure or lumped-parameter models, and their effects on simulation results.

The vascular resistances in the arterioles and capillaries are largely responsible for producing the blood pressures observed in the large arteries. The same vascular resistances are also responsible for regulating the distribution of blood flow into different regions of the body. A fluid dynamic simulation of large arteries alone, without consideration of the smaller downstream vessels, neglects this important effect of vascular resistance.

Figure 3a shows an example of such a scenario, where a zero pressure outflow boundary condition is used. The pressure in the 3D domain is much lower than normal physiological levels, and the amount of flow through the two different outlets is solely determined by the vessel geometry. Without any downstream resistances, small variations in the conduit resistance in the 3D domain lead to drastic flow bias. In the example shown, the average flow rates through the main and branch outlet are $43 \mathrm{~mL} / \mathrm{s}$ and $2 \mathrm{~mL} / \mathrm{s}$, respectively, meaning that almost no flow is passing through the branch outlet. Also, as a result of near zero pressure, the outlet flows are artificially sensitive to small pressure perturbations in the 3D domain from accelerations of fluid. This artifact is seen in the example as the branch outlet exhibiting reverse flow during the deceleration phase of the inflow waveform. This is inconsistent with most physiological scenarios where the larger branch is more likely to exhibit reverse flow. Due to artifacts and the lack of ability for adjustments, the resulting pressure, flow, and flow split from using a zero pressure boundary condition usually cannot reflect realistic physiological conditions.

Resistance outflow boundary condition is a simple approach to partially capture the effects of downstream vasculature, thereby achieving appropriate flow split between the outlets. Figure $3 \mathrm{~b}$ shows an example simulation with the same 3D geometry as $3 \mathrm{a}$ but with resistance outflow boundary condition. The ratio between the resistances at the two outlets was adjusted to vary the flow split; the average flow rates through the main and branch outlet are now $24 \mathrm{~mL} / \mathrm{s}$ and $21 \mathrm{~mL} / \mathrm{s}$, respectively. The flows through the outlets are now governed principally by the 
outflow resistances and no longer by small resistance and pressure perturbations in the 3D domain. Note that with resistance outlet boundary condition prescription, there is a direct linear relationship between the flow and pressure. The pressure waveform, as well as the flow waveforms through the two outlets, all have the same shape and no phase-lag relative to each other. More importantly, this direct linear relationship can lead to large swings of pressure as flow varies over time. In the example shown, the average pressure over the cardiac cycle is 112 $\mathrm{mmHg}$, which is physiologically realistic, but the pressure peak is unrealistically high due to the pulsatile inflow. In summary, resistance outflow boundary conditions can be useful in steady or near-steady flow cases, but for pulsatile flow conditions, a more complicated lumped-parameter model outflow boundary condition should be used.

Due to blood vessel compliance, wave propagation occurs in the vasculature, leading to pressure and flow waveforms with different shapes and phases. Outflow boundary condition prescription using a Windkessel model containing a capacitance element can account for the downstream vessel compliance and add another level of realism to the simulation. Using the same outlet resistances as in Fig 3b, we now add in a capacitance element to observe the effect in Fig $3 \mathrm{c}$. The pressure and flows through the outlets averaged across the cardiac cycle $(112 \mathrm{mmHg}, 24 \mathrm{~mL} / \mathrm{s}$ through main outlet, $21 \mathrm{~mL} / \mathrm{s}$ through branch outlet) are the same as in $3 \mathrm{~b}$, but we can see that there are now waveform shape and phase differences between pressure and flow, as well as between flows through the different outlets. In addition, the energy storage of the capacitor allows the pressure oscillation to be dampened and results in more realistic pressure peaks. This enables greater flexibility for the simulation to capture desired physiological scenarios.

The momentum of the flowing blood presents another variable that affects the relationship between pressure and flow. We can capture the blood momentum effect in the downstream vasculature by incorporating inductance elements in the outflow boundary Windkessel model. Keeping the same outlet resistance and capacitance values, Figure $3 \mathrm{~d}$ shows an example simulation with Windkessel outflow boundary conditions now containing inductances. Note that there is an additional phase difference between pressure and flow due to the inductance elements. Compared to Fig $3 \mathrm{c}$, the pressure waveform is shifted forward in time and now peaks before the flow peak. The phase difference between the outlet flows can also be adjusted via the ratio of the outlet inductance values. In a practical situation the inductance offers another degree of adjustment to achieve correct pressure and flow phase differences.

Using resistance, capacitance, and inductance elements in lumped-parameter circuits, we can fully capture the important relevant phenomena occurring in the downstream vasculature and achieve realistic boundary condition prescription for the fluid dynamic simulation.

\section{Modeling the circulation with a lumped parameter network}

Here we present the mathematical formulations for some of the aforementioned lumpedparameter boundary conditions, as well as for a heart and a coronary circulation model. In lumped parameter networks, we "lump" the resistive, elastic and inertial properties of blood flow through vessels into electrical elements. Therefore, our circulation model becomes analogous to an electric circuit. We then solve the associated set of ordinary differential equations (ODEs) governing the electrical circuit. The values of circuit elements are tuned to match physiologically realistic values for flow, pressure, and other quantities.

We use the basic circuit element relations defining a resistor, capacitor and inductor, with appropriate analogies to fluid dynamics as follows: 


$$
\begin{gathered}
V=I R \Leftrightarrow \Delta P=Q R \text { (resistor) } \\
V=\frac{d}{d t} L I \Leftrightarrow \Delta P=\frac{d}{d t} L Q \text { (inductor) } \\
I=\frac{d}{d t} C V \Leftrightarrow Q=\frac{d}{d t} C P \text { (capacitor) }
\end{gathered}
$$

Blood viscosity acts to resist flow for a given pressure drop so that, from above,

$$
Q=\frac{\Delta P}{R}
$$

hence increasing resistance leads to a lower flow rate for a fixed pressure gradient. We recall that for Poiseulle flow, resistance to flow is

$R=\frac{8 \mu l}{\pi a^{4}}$

where $a$ is the vessel radius and $l$ is the vessel length, so that resistance drops dramatically with increasing vessel radius.

\subsection{Windkessel models}

A basic lumped model is a simple 3-element Windkessel model containing two resistors and a capacitor. This model accounts for the energy loss due to viscosity and energy storage due to vessel distensibility of the vasculature. Windkessel in German means "air chamber," but is generally taken to imply an elastic reservoir. In the realm of the cardiovascular system, the vessels act as this elastic reservoir.

Applying Kirchhof's current law (conservation of mass) to the 3-element model shown in Figure 4, we have

$Q=Q_{r}=Q_{C}+Q_{R}$

and the element relations are given by

$Q_{c}=c \frac{d p_{d}}{d t}$

$p-p_{d}=Q r$

$p_{d}=Q_{R} R$

where $p-p_{d}$ is the pressure drop across the proximal resistor. The above relations can be combined into a single ODE, together with conservation of mass, and time constant $\tau=R C$ to obtain 
$\frac{d p}{d t}+p \frac{1}{\tau}=r \frac{d Q}{d t}+\frac{1}{\tau}(r+R) Q$

which has solution

$p(t)=e^{-\frac{t}{\tau}}\left[\int_{0}^{t} e^{\frac{s}{\tau}}\left[r \frac{d Q(s)}{d s}+\frac{1}{\tau}(r+R) Q(s)\right] d s+p(0)\right]$.

Referring again to Figure 2, we note that the RCR circuit can be used as a boundary condition for large vessels such as the aorta, and branch vessels going to head and neck. In this case, the RCR circuit models the proximal and distal resistance and capacitance of the vasculature distal to the outlet of the model.

The four-element Windkessel model incorporates the inertia of blood in the proximal circulation by adding an inductor to the 3 element model. Conservation of mass for this circuit implies

$Q=Q_{r}=Q_{L}=Q_{C}+Q_{R}$

and the element relations are given as

$p-p_{d}=L \frac{d Q_{L}}{d t}+Q_{r} r_{,}$

$Q_{C}=c \frac{d p_{d}}{d t}$

$p_{d}=Q_{R} R$,

Continuity combined with the last two element relations produces the ODE

$\frac{d p_{d}}{d t}+\frac{p_{d}}{R C}=\frac{Q}{C}$

Continuity combined with the first element relation produces:

$p=L \frac{d Q}{d t}+Q r+p_{d}$

which can then be solved using the solution from the first ODE.

\subsection{A Sample Heart Model}

A simple ventricle model can be assembled using the resistor, capacitor, and inductor components described above, with the addition of a diode to represent the heart valve and an elastance function to model ventricular contraction, as shown in Figure 5. Given variables $P_{u}, L_{u}, R_{d}, E_{d}, V_{d 0}$, we model the inductor with

$\frac{d Q_{v}}{d t}=\frac{1}{L_{u}}\left(P_{u}-P_{d}\right)$

the capacitor with 
$P_{d}=\frac{1}{C_{d}}\left(V_{d}-V_{d 0}\right)$,

where $V_{d 0}$ is the volume of the downstream chamber at zero pressure, and the resistor with

$$
P_{d}=Q_{d} R_{d}
$$

We require that flow goes only one direction through the valve, such that we must have $P_{u}>P_{d}$

for an open valve. We wish to derive a set of ODEs to govern this model. We start by relating pressure to elastance by

$P_{d}=E_{d}(t)\left[V_{d}-V_{d 0}\right]$

Substituting this into the inductor equation above we have

$\frac{d Q_{v}}{d t}=\frac{1}{L_{u}}\left\{P_{u}-E_{d}(t)\left[V_{d}-V_{d 0}\right]\right\}$

and into the equation for resistance we have

$Q_{d}=\frac{E_{d}\left[V_{d}-V_{d 0}\right]}{R_{d}}$.

The difference in flow through the valve and downstream is

$\frac{d V_{d}}{d t}=Q_{v}-Q_{d}=Q_{v}-\frac{E_{d}\left(V_{d}-V_{d 0}\right)}{R_{d}}$

from the equation above. This results in two ODEs for ventricular volume and flow

$\frac{d Q_{v}}{d t}=\frac{1}{L_{u}}\left\{P_{u}-E_{d}\left[V_{d}-V_{d 0}\right]\right\}$

$\frac{d V_{d}}{d t}=Q_{v}-\frac{E_{d}\left(V_{d}-V_{d 0}\right)}{R_{d}}$

which can be solved for two scenarios: 1) valve closed such that $P_{d} \geq P_{u}$ and $Q_{v}=0$, and 2)

valve open such that $P_{u}>P_{-} d$ and we evaluate $Q_{v^{*}}$

\subsection{A Coronary Artery Lumped Model}

The coronary arteries are particularly challenging to model due to the interaction of coronary resistance with heart contraction. Coronary flow peaks during diastole, when aortic flow is at a minimum, because coronary vascular resistance is reduced with ventricular relaxation. On the other hand, during systole, coronary resistance is high due to ventricular contraction, and coronary flow is at a minimum. This is particularly pronounced in the left coronary artery. A lumped model for use as a coronary artery boundary condition was proposed by Kim et al and is shown in Figure 6.[43, 44] This circuit is governed by the following second order ODE, 
$p_{2} \frac{d^{2} P}{d t^{2}}+p_{1} \frac{d P}{d t}+p_{0} P=q_{2} \frac{d^{2} Q}{d t^{2}}+q_{1} \frac{d Q}{d t}+q_{0} Q+b_{1} \frac{d P_{\text {i } m}}{d t}$

where $P(t)$ and $Q(t)$ are the time varying pressure and flow, the constants are functions of the resistances and compliances of the lumped model, and $P_{\text {im }}(t)$ is the intramyocardial pressure.

This ODE has analytic solution

$$
\begin{gathered}
P(t)=\left(R Q(t)+\int_{0}^{t} e^{\lambda_{1}(t-s)} Z_{1} Q(s) d s\right)-\int_{0}^{t} e^{\lambda_{2}(t-s)} Z_{2} Q(s) d s+\left(A e^{\lambda_{1} t}-B e^{\lambda_{2} t}\right) \\
+\left(\int_{0}^{t} e^{\lambda_{1}(t-s)} Y_{1} P_{\text {i } m}(s) d s-\int_{0}^{t} e^{\lambda_{2}(t-s)} Y_{2} P_{\text {i } m}(s) d s\right)
\end{gathered}
$$

where the constants are obtained from coefficients of the ODE and initial conditions, as described previously by Kim et al. Now directing our attention back to Figure 2, we observe that these coronary lumped models can be coupled to a 3D model. Because there is a known analytic solution to this ODE, coupling can be done monolithically using the multidomain method introduced by Vignon et al, and following the work of Kim et al.[2, 43]

\section{Modeling the circulation with the 1-D equations of Blood Flow}

An alternative to the lumped parameter approach is to solve the one-dimensional equations of blood flow, which offers a compromise between full 3D models and 0D lumped-parameter networks with no spatial dimension. Derivations of the 1-D equations can be found in previous work, along with details of coupling algorithms.[14]

\section{Closed-loop Multi-scale Simulations}

To capture the interaction between the 3D domain and the circulatory system, it is necessary to couple 3D simulations to reduced order models such as 1-D equations or lumped-parameter models that capture circulatory dynamics.[45] The open loop lumped-parameter models described above can be expanded to full closed-loop networks that can then be coupled to the 3D domain.[3, 46-48]

A closed-loop lumped-parameter model has the advantage that the effects from all parts of the circulation are fully coupled together to influence the overall simulated physiology. Figure 7 shows an example of a closed-loop multi-scale simulation setup. Compared to the open-loop examples presented in figure 3, there is no longer any prescribed fixed inflow to the 3D domain, but rather the boundary conditions are results of solving the $0 \mathrm{D}$ model equations, which describe the responses of the heart as well as different parts of the circulation to pressure and flow. We have discussed the importance of the boundary conditions provided by reduced-order models in a simulation. In a closed-loop scenario, the 3D domain presents itself to the $0 \mathrm{D}$ model as a timevarying resistance, inductance, and capacitance (in the case of deformable wall simulation) that are functions of the flow patterns within the 3D domain. The 0D model behavior is affected by 
this 3D model behavior, and thus provides boundary conditions that are fully coupled to the 3D domain, forming a complete feedback loop.

Closed-loop multi-scale models have been particularly useful in modeling coronary flows, as well as complex surgeries in pediatric cardiology applications[3]. For example, the effects of a surgical shunt in single ventricle patients can be investigated using such a model, which enables considerations of how the shunt resistance influences the balance of blood flow through various pathways in light of other systemic resistances and heart behavior[47, 49, 50].

\section{Example Case Studies}

\subsection{Virtual Surgeries of the Superior Cavopulmonary Connection}

In this case study we demonstrate the application of patient-specific computational modeling to the virtual planning of surgical procedures. We demonstrate the power and potential of modeling to predict vascular surgical outcomes and obtain detailed hemodynamic information at zero risk to the patient, through a case study of a patient-specific model of a single ventricle stage 2 procedure.

Single ventricle congenital defects result in a heart that has only one functional pumping chamber. Palliation for these conditions typically requires a three-staged operative process, where a shunt is placed between the systemic and pulmonary arteries in stage 1, and the superior venous return is connected to the pulmonary circulation in stage 2 . The stage 2 palliative surgery has two options: the "Glenn" or "Hemi-Fontan" procedures[51]. The Glenn procedure involves directly connecting the superior vena cava to the right pulmonary artery, and the Hemi-Fontan procedure involves reconstruction of the right atrium and pulmonary artery to allow for an enlarged confluence with the superior vena cava. Various degrees of pulmonary stenosis are also common among these patients, and additional surgical steps are often taken to remove the stenosis during the stage 2 procedure. Using a multi-scale blood flow simulation, we can computationally investigate the hemodynamic differences between the Glenn and the HemiFontan surgical options, as well as the necessity of pulmonary stenosis (if one is present) palliation during the stage 2 surgery.

The computational model is created using the pre-operative imaging and clinical data acquired in the patient. From the patient imaging data, we can construct a pre-operative 3D model that is a representation of the patient's pre-operative anatomy. Then based on the preoperative 3D model, we perform "virtual" Glenn or Hemi-Fontan surgeries by computationally modifying the pre-operative 3D model. Additionally, we add a "virtual" left pulmonary stenosis, to create different 3D models representing various stage 2 scenarios (Figure 8). Using the preoperative clinical data, we also tune the closed-loop lumped-parameter network (0D model) to represent the systemic circulation of the patient, and couple the network to the 3D model to complete a multi-scale simulation setup (Figure 9).

Using commercial mesh generation software (MESHSIM, Simmetrix, Inc., NY), the 3D models are discretized into isotropic finite-element meshes with a maximum edge size of $0.3 \mathrm{~mm}$ and containing 864,000 1.3 million linear tetrahedral elements. We couple the 3D anatomical model to the lumped-parameter network, and numerically compute the coupled system using custom stabilized finite-element method solving the incompressible Navier-Stokes equations (Simvascular, www.simtk.org) while assuming non-moving vessel walls.[45, 52] Neumann boundary conditions are applied between the 3D and 0D models, in which the 3D model receives 
pressure information from the $0 \mathrm{D}$ model, and returns flow information to the $0 \mathrm{D}$ model. Flow and pressure in the 0D model are computed from a set of algebraic and ordinary differential equations derived from the lumped-parameter network, using a 4th order Runge-Kutta method.

We extract clinically relevant information from the results of these multi-scale simulations, such as the 3D pressure and flow in the anatomical model (Figure 10), time tracings of various quantities in the circulation (Figure 11), measures of power loss in the surgical junction relative to other systemic powers (Figure 12), etc. From the results of this case study, we see that the different surgical options and pulmonary palliation would not greatly affect the physiologies at the systemic level. However, local fluid dynamics differences do exist in the surgical junction, and the 3D hemodynamics data can be used to consider clinical issues such as thrombotic risk. In addition, the analysis of power loss within the surgical junction relative to systemic power can be used to provide pulmonary stenosis palliation decision support. In this particular example case and under the physiology investigated, it appears that a $50 \%$ or $85 \%$ area stenosis would not introduce a significant load to the cardiovascular system as a whole, nor significantly impact overall physiology. The results extracted from these simulations suggest that hemodynamic differences between the surgical options for this particular patient may be small, and that surgical considerations could place higher priorities to other factors such as procedural risk or the confidence of the surgeon towards different procedures.

\subsection{Thrombotic Risk in Kawasaki Disease Aneurysms}

In this example we show how blood flow simulation is applied to investigate hemodynamics in coronary artery aneurysms caused by Kawasaki disease (KD), and how they differ from the hemodynamics in healthy coronary arteries.[4]

$\mathrm{KD}$ is an acute vasculitis occurring primarily in young children. The acute phase of the disease can damage the coronary arteries and leave coronary aneurysms in approximately $25 \%$ of untreated cases[53], and 3-5\% of intravenous immunoglobulin treated cases[54]. These coronary aneurysms elevate the risk of thrombosis, which can then lead to myocardial infarction and sudden death[55]. Without a thrombotic episode, patients with KD coronary aneurysms are typically asymptomatic, and patient selection for treatments such as systemic anticoagulation and coronary artery bypass surgery[56] is challenging. Hemodynamic parameters from blood flow simulations may ultimately provide an estimate for the risk of thrombosis and help guide patient management.

Based on clinical imaging data from a 10-year-old patient who had suffered KD at the age of 3, and developed giant coronary artery aneurysms, we constructed a 3D computational model of the patient anatomy (Figure 13a). Another 3D model was created to represent a normal healthy coronary artery anatomy by replacing the aneurysmal regions with normal coronary geometry (Figure 13b). This allows for a direct comparison of hemodynamic differences between the normal and pathological states of the same patient. We construct a finite element mesh for each model using commercial software (Symmetrix, Inc, Troy, NY). Adaptive meshing with a minimum mesh size of $0.2 \mathrm{~mm}$ is then applied based on the Hessian of the velocity field. The resulting meshes have over 3.5 million elements for both the normal and the KD model.

Figure 14 represents the multi-scale simulation setup using these $3 \mathrm{D}$ anatomical models coupled to reduced-order 0D models. We prescribe an inflow boundary condition at the aortic root, coronary boundary conditions at the coronary artery outlets, and RCR boundary conditions at all other outlets of the model. Readers may refer to the previous sections for detailed descriptions of these types of boundary conditions. The parameters in the 0D models are tuned 
using a combination of clinical data measured in the patient, and literature knowledge of flow and resistances. Computation of the coupled system is then performed using the methods described in the previous case study.

From simulated pressure and flow results, we compute wall shear stress and particle residence times. Figure 15 compares the spatially averaged WSS between the KD and normal cases at different locations. It is clear that the aneurysmal coronary arteries exhibit much lower wall shear stresses compared to the normal. Particle-tracking analysis also demonstrates differences between normal and aneurysmal coronaries. In the aneurysmal case $30 \%$ of particles released at the aortic root circulate in the aneurysms for one full cardiac cycle, and some particles remain in the domain for as long as 5 cardiac cycles. In the normal coronary artery model, all particles exit the domain within 1.3 cardiac cycles. With continual improvement of understandings of how hemodynamic parameters specifically correlate with thrombotic risk, these blood flow simulation results will provide increasingly valuable information towards clinical support of KD patient management.

\subsection{Hepatic Blood Flow Distribution in the Fontan Junction}

In this case study, we demonstrate how blood flow simulations are used to evaluate the performance of different Fontan surgical geometries, using hepatic flow distribution to each lung as a metric of performance.[57]

As presented in section 8.1, palliation for single ventricle defects typically requires a threestaged operative process. Here we focus on the stage-3 Fontan completion procedure, in which the inferior vena cava (IVC) is connected to the pulmonary arteries, restoring an in-series systemic-to-pulmonary circulation. One clinical consideration regarding this physiology is the development of pulmonary arteriovenous malformations, which is known to correlate with hepatic flow distribution, meaning the fractional split of the blood returning from the liver through the IVC entering each lung. Studies have revealed a clear link between the absence of hepatic venous return and the development of pulmonary arteriovenous malformations, suggesting that the hepatic blood flow may contain factors required for proper lung development.[58] It is therefore important to consider the maintainance of an even hepatic flow distribution while designing a Fontan surgical geometry, to ensure the proper development of both lungs.

There are various surgical options for the stage 3 procedure, including the $\mathrm{T}$-junction option where the IVC is anastomosed to the pulmonary arteries via a straight vascular graft, the offset option where a curved graft is anastomosed to the left pulmonary artery, and the Y-graft option where a bifurcated $\mathrm{Y}$-shaped graft is anastomosed to the left and right pulmonary arteries separately.[59, 60] For each surgical option, there can be also variations in the geometrical design.

In this case study we evaluate the performance of various surgical alternatives in terms of hepatic distribution in 5 patients. Starting from a pre-operative 3D anatomical model constructed based on clinical imaging data of each patient, we perform virtual stage 3 surgeries to construct various Fontan surgical junction geometries based on different surgical alternatives (Figure 16). Following common clinical practice, the virtual surgeries adapt $\mathrm{Y}$-grafts with a 20 -mm diameter trunk and 15-mm diameter branches, and T-junction and offset grafts with a 20 -mm diameter trunk. The anatomical models are then processed via isotropic tetrahedral mesh generation and anisotropic mesh adaptation, resulting in final finite element meshes consisting of approximately 1 to 1.5 million elements. 
Based on pre-operative phase-contrast MRI acquired in each patient, we prescribe inflow boundary conditions to the superior vena cava (SVC) and IVC faces of each anatomical model. Due to the anatomical differences pre and post surgery affecting the IVC flow waveform shape, the amplitude of the IVC flow waveform is scaled to match that of a typical Fontan patient. For the pulmonary outlet branches, we prescribe RCR boundary conditions tuned to pre-operative pressure and flow split, assuming that they do not change in the immediate post-operative scenario.

From the 3D simulation results, we perform Lagrangian particle tracking to quantify the hepatic flow distribution. Massless particles are computationally released at the IVC face and tracked until they exit the domain through a pulmonary outlet (Figure 17). The fraction of IVC flow that enters the left and right pulmonary arteries for different surgical alternatives for each patient is then obtained and shown in Figure 18. The theoretical optimum hepatic distribution is calculated based on conservation of mass and the total amount of flow entering each lung.

We can see from these results that the different surgical alternatives can indeed return drastically different hepatic distribution performances in each patient. Surgical alternatives can be selected with these considerations in mind. The blood flow simulation analyses presented in this case study provide means to predict surgical outcomes and to aid in surgical decision support for the Fontan stage 3 procedure.

\section{Future Perspective}

Simulation technology for cardiovascular disease has now advanced to the point of successful clinical adoption in select studies, with more widespread adoption likely to follow in the near future. Most notably, simulations led to the development and clinical pilot studies of the Fontan Y-graft surgery, and recent favorable comparisons of simulation-derived fractional flow reserve (FFR_CT) with catheter measurements in adult coronary artery disease patients.[61]

There are several roadblocks currently preventing wider adoption of simulation tools and application to large-scale clinical studies. First, there is a pressing need for clinical validation data and statistical correlation with patient outcomes. Unlike other traditional engineering fields, acquisition of in vivo validation data is often hindered by ethical considerations and imaging limitations. However, this is likely the single most important factor currently hindering the adoption of simulation methods in the clinic. Second, there is a need for a numerical framework to handle uncertainty propagation from simulation inputs (e.g. clinical data and noisy images) to outputs (e.g. simulation predictions of patient risk). Together with uncertainties, parameter identification should be used to automatically tune models to match available clinical data in the presence of uncertainties. Third, there is a pressing need for accelerated image segmentation tools to automate the model construction process. Without these tools to reduce laborious userinput, simulations will remain limited to small-scale clinical studies.

In addition to overcoming the above roadblocks, there are several important new areas of research that will enhance the future scientific and clinical value of cardiovascular simulations. First, there is a need to couple simulations with models of vascular growth and remodeling. This would enable fluid solid growth simulations, in which vessels would adapt in silico to changing flow and pressure conditions. Second, there is a need to couple simulations with biochemical models of the thrombosis process. This would allow for improved prediction of thrombotic risk due to aneurysms, for stroke patients, or inside medical devices. Finally, fluid structure interaction simulations should be improved to fully model the vessel wall, including variable properties and external tissue support, and characterization of patient specific material properties. 


\section{References}

1. Figueroa CA, Vignon-Clementel IE, Jansen KE, Hughes TJR, Taylor CA: A coupled momentum method for modeling blood flow in three-dimensional deformable arteries. Computer Methods in Applied Mechanics and Engineering 2006, 195(4143):5685-5706.

2. Vignon-Clementel IE, Figueroa CA, Jansen KE, Taylor CA: Outflow boundary conditions for three-dimensional finite element modeling of blood flow and pressure in arteries. Computer Methods in Applied Mechanics and Engineering 2006, 195(2932):3776-3796.

3. Sankaran S, Moghadam ME, Kahn AM, Tseng EE, Guccione JM, Marsden AL: PatientSpecific Multiscale Modeling of Blood Flow for Coronary Artery Bypass Graft Surgery. Annals of Biomedical Engineering 2012, 40(10).

4. Sengupta D, Kahn AM, Burns JC, Sankaran S, Shadden SC, Marsden AL: Image-based modeling of hemodynamics in coronary artery aneurysms caused by Kawasaki disease. Biomechanics and Modeling in Mechanobiology 2012, 11(6):915-932.

5. Womersley JR: Mathematical theory of oscillating flow in an elastic tube. The Journal of physiology 1955, 127(2):37-38P.

6. Womersley JR: METHOD FOR THE CALCULATION OF VELOCITY, RATE OF FLOW AND VISCOUS DRAG IN ARTERIES WHEN THE PRESSURE GRADIENT IS KNOWN. Journal of Physiology-London 1955, 127(3):553-563.

7. Womersley JR: OSCILLATORY MOTION OF A VISCOUS LIQUID IN A THINWALLED ELASTIC TUBE .1. THE LINEAR APPROXIMATION FOR LONG WAVES. Philosophical Magazine 1955, 46(373):199-221.

8. Womersley JR: OSCILLATORY FLOW IN ARTERIES - THE CONSTRAINED ELASTIC TUBE AS A MODEL OF ARTERIAL FLOW AND PULSE TRANSMISSION. Physics in Medicine and Biology 1957, 2(2):178-187.

9. Womersley JR: OSCILLATORY FLOW IN ARTERIES .2. THE REFLECTION OF THE PULSE WAVE AT JUNCTIONS AND RIGID INSERTS IN THE ARTERIAL SYSTEM. Physics in Medicine and Biology 1958, 2(4):313-323.

10. Womersley JR: OSCILLATORY FLOW IN ARTERIES .3. FLOW AND PULSEVELOCITY FORMULAE FOR A LIQUID WHOSE VISCOSITY VARIES WITH FREQUENCY. Physics in Medicine and Biology 1958, 2(4):374-382.

11. Thiry PS, Roberge FA: ANALOGS AND MODELS OF SYSTEMIC ARTERIAL BLOOD-FLOW. Revue Canadienne De Biologie 1976, 35(4):217-238.

12. Westerho.N, Bosman F, Devries CJ, Noorderg.A: ANALOG STUDIES OF HUMAN SYSTEMIC ARTERIAL TREE. Journal of Biomechanics 1969, 2(2):121-\&.

13. Westerhof N, Lankhaar JW, Westerhof BE: The arterial Windkessel. Medical \& Biological Engineering \& Computing 2009, 47(2):131-141.

14. Hughes TJR, Lubliner J: ON THE 1 DIMENSIONAL THEORY OF BLOOD FLOW IN THE LARGER VESSELS. Mathematical Biosciences 1973, 18(1-2):161-170.

15. Formaggia L, Gerbeau JF, Nobile F, Quarteroni A: On the coupling of 3D and 1D Navier-Stokes equations for flow problems in compliant vessels. Computer Methods in Applied Mechanics and Engineering 2001, 191(6-7):561-582.

16. Taylor CA, Hughes TJR, Zarins CK: Finite element modeling of blood flow in arteries. Computer Methods in Applied Mechanics and Engineering 1998, 158(1-2):155-196.

17. Taylor CA, Hughes TJR, Zarins CK: Finite element modeling of three-dimensional 
pulsatile flow in the abdominal aorta: Relevance to atherosclerosis. Annals of Biomedical Engineering 1998, 26(6):975-987.

18. Taylor CA DM, Ku JP, Parker D, Steele BN, Wang K, Zarins CK: Predictive Medicine: Computational Techniques in Therapeutic Decision-Making. Computer Aided Surgery 1999, 4:231-247.

19. Milner JS, Moore JA, Rutt BK, Steinman DA: Hemodynamics of human carotid artery bifurcations: Computational studies with models reconstructed from magnetic resonance imaging of normal subjects. In: 1998; 1998: 143-156.

20. Steinman DA: Image-based computational fluid dynamics modeling in realistic arterial geometries. Annals of Biomedical Engineering 2002, 30(4):483-497.

21. Castro MA, Putman CM, Cebral JR: Patient-specific computational fluid dynamics modeling of anterior communicating artery aneurysms: A study of the sensitivity of intra-aneurysmal flow patterns to flow conditions in the carotid arteries. American Journal of Neuroradiology 2006, 27(10):2061-2068.

22. Whitehead KK, Pekkan K, Kitajima HD, Paridon SM, Yoganathan AP, Fogel MA: Nonlinear power loss during exercise in single-ventricle patients after the Fontan Insights from computational fluid dynamics. Circulation 2007, 116(11):I165-I171.

23. Gijsen FJH, van de Vosse FN, Janssen JD: The influence of the non-Newtonian properties of blood on the flow in large arteries: steady flow in a carotid bifurcation model. Journal of Biomechanics 1999, 32(6):601-608.

24. Malladi R, Sethian JA, Vemuri BC: SHAPE MODELING WITH FRONT PROPAGATION - A LEVEL SET APPROACH. Ieee Transactions on Pattern Analysis and Machine Intelligence 1995, 17(2):158-175.

25. Malladi R, Sethian JA, Vemuri BC: A fast level set based algorithm for topologyindependent shape modeling. Journal of Mathematical Imaging and Vision 1996, 6(23):269-289.

26. Wang K: Level Set Methods for Computational Prototyping with Application to Hemodynamic Modeling. Stanford, CA: Stanford University; 2001.

27. Dollár P, Tu Z, Belongie S: Supervised Learning of Edges and Object Boundaries. In: CVPR. New York City; 2006.

28. Hughes TJR, Mallet M, Mizukami A: A NEW FINITE-ELEMENT FORMULATION FOR COMPUTATIONAL FLUID-DYNAMICS .2. BEYOND SUPG. Computer Methods in Applied Mechanics and Engineering 1986, 54(3):341-355.

29. Peiro J, Sherwin SJ, Giordana S: Automatic reconstruction of a patient-specific highorder surface representation and its application to mesh generation for CFD calculations. Medical \& Biological Engineering \& Computing 2008, 46(11):1069-1083.

30. Sherwin SJ, Karniadakis GE: Tetrahedral spectral elements for CFD. In: Fourteenth International Conference on Numerical Methods in Fluid Dynamics Proceedings of the Conference. edn. Edited by Deshpande SM, Desai SS, Narasimha R: Springer-Verlag; 1995: 429-434434.

31. Peskin CS: FLOW PATTERNS AROUND HEART VALVES - NUMERICAL METHOD. Journal of Computational Physics 1972, 10(2):252-\&.

32. Peskin CS: NUMERICAL-ANALYSIS OF BLOOD-FLOW IN HEART. Journal of Computational Physics 1977, 25(3):220-252.

33. Zheng X, Seo JH, Vedula V, Abraham T, Mittal R: Computational modeling and analysis of intracardiac flows in simple models of the left ventricle. European Journal 
of Mechanics B-Fluids 2012, 35:31-39.

34. Valen-Sendstad K, Mardal K-A, Mortensen M, Reif BAP, Langtangen HP: Direct numerical simulation of transitional flow in a patient-specific intracranial aneurysm. Journal of Biomechanics 2011, 44(16):2826-2832.

35. Ford MD, Piomelli U: Exploring High Frequency Temporal Fluctuations in the Terminal Aneurysm of the Basilar Bifurcation. Journal of Biomechanical Engineering-Transactions of the Asme 2012, 134(9).

36. Long CC, Hsu MC, Bazilevs Y, Feinstein JA, Marsden AL: Fluid-structure interaction simulations of the Fontan procedure using variable wall properties. Communications in Numerical Methods in Engineering 2012, 28(5).

37. Bazilevs Y, Hsu MC, Zhang Y, Wang W, Kvamsdal T, Hentschel S, Isaksen JG: Computational vascular fluid-structure interaction: methodology and application to cerebral aneurysms. Biomechanics and Modeling in Mechanobiology 2010, 9(4):481498.

38. Kung EO, Les AS, Figueroa CA, Medina F, Arcaute K, Wicker RB, McConnell MV, Taylor CA: In Vitro Validation of Finite Element Analysis of Blood Flow in Deformable Models. Annals of Biomedical Engineering 2011, 39(7):1947-1960.

39. Balossino R, Pennati G, Migliavacca F, Formaggia L, Veneziani A, Tuveri M, Dubini G: Computational models to predict stenosis growth in carotid arteries: Which is the role of boundary conditions? Computer Methods in Biomechanics and Biomedical Engineering 2009, 12(1):113-123.

40. Vignon IE, Taylor CA: Outflow boundary conditions for one-dimensional finite element modeling of blood flow and pressure waves in arteries. Wave Motion 2004, 39(4):361-374.

41. Duan B, Zamir M: APPROXIMATE SOLUTION FOR PULSATILE FLOW IN TUBES OF SLIGHTLY NONCIRCULAR CROSS-SECTIONS. Utilitas Mathematica 1991, 40:13-26.

42. Hodis S, Zamir M: Mechanical events within the arterial wall under the forces of pulsatile flow: A review. Journal of the Mechanical Behavior of Biomedical Materials 2011, 4(8):1595-1602.

43. Kim HJ, Vignon-Clementel IE, Figueroa CA, Jansen KE, Taylor CA: Developing computational methods for three-dimensional finite element simulations of coronary blood flow. Finite Elements in Analysis and Design 2010, 46(6):514-525.

44. Kim HJ, Vignon-Clementel IE, Coogan JS, Figueroa CA, Jansen KE, Taylor CA: Patient-Specific Modeling of Blood Flow and Pressure in Human Coronary Arteries. Annals of Biomedical Engineering 2010, 38(10):3195-3209.

45. Moghadam ME, Vignon-Clementel IE, Figliola R, Marsden AL, Modeling Congenital Hearts A: A modular numerical method for implicit 0D/3D coupling in cardiovascular finite element simulations. Journal of Computational Physics 2013, 244:63-79.

46. Baretta A, Corsini C, Yang W, Vignon-Clementel IE, Marsden AL, Feinstein JA, Hsia TY, Dubini G, Migliavacca F, Pennati G et al: Virtual surgeries in patients with congenital heart disease: a multi-scale modelling test case. Philosophical Transactions of the Royal Society a-Mathematical Physical and Engineering Sciences 2011, 369(1954):4316-4330.

47. Corsini C, Cosentino D, Pennati G, Dubini G, Hsia T-Y, Migliavacca F: Multiscale 
models of the hybrid palliation for hypoplastic left heart syndrome. Journal of Biomechanics 2011, 44(4):767-770.

48. Kung E, Baretta A, Baker C, Arbia G, Biglino G, Corsini C, Schievano S, VignonClementel IE, Dubini G, Pennati G et al: Predictive modeling of the virtual HemiFontan operation for second stage single ventricle palliation: Two patient-specific cases. Journal of Biomechanics 2013, 46(2):423-429.

49. Moghadam ME, Migliavacca F, Vignon-Clementel IE, Hsia T-Y, Marsden AL, Modeling Congenital Hearts A: Optimization of Shunt Placement for the Norwood Surgery Using Multi-Domain Modeling. Journal of Biomechanical Engineering-Transactions of the Asme 2012, 134(5).

50. Migliavacca F, Balossino R, Pennati G, Dubini G, Hsia TY, de Leval MR, Bove EL: Multiscale modelling in biofluidynamics: Application to reconstructive paediatric cardiac surgery. Journal of Biomechanics 2006, 39(6):1010-1020.

51. Norwood JWI: Hypoplastic left heart syndrome. The Annals of Thoracic Surgery 1991, 52(3):688 - 695.

52. Schmidt JP, Delp SL, Sherman MA, Taylor CA, Pande VS, Altman RB: The Simbios National Center: Systems biology in motion. Proceedings of the Ieee 2008, 96(8):1266-1280.

53. Kato H, Sugimura T, Akagi T, Sato N, Hashino K, Maeno Y, Kazue T, Eto G, Yamakawa R: Long-term consequences of Kawasaki disease - A 10- to 21-year follow-up study of 594 patients. Circulation 1996, 94(6):1379-1385.

54. NEWBURGER J, TAKAHASHI M, BEISER A, BURNS J, BASTIAN J, CHUNG K, COLAN S, DUFFY C, FULTON D, GLODE M et al: A SINGLE INTRAVENOUSINFUSION OF GAMMA-GLOBULIN AS COMPARED WITH 4 INFUSIONS IN THE TREATMENT OF ACUTE KAWASAKI SYNDROME. New England Journal of Medicine 1991, 324(23):1633-1639.

55. Gordon J, Kahn A, Burns J: When Children With Kawasaki Disease Grow Up Myocardial and Vascular Complications in Adulthood. Journal of the American College of Cardiology 2009, 54(21):1911-1920.

56. Newburger JW, Takahashi M, Gerber MA, Gewitz MH, Tani LY, Burns JC, Shulman ST, Bolger AF, Ferrieri P, Baltimore RS et al: Diagnosis, treatment, and long-term management of Kawasaki disease: a statement for health professionals from the Committee on Rheumatic Fever, Endocarditis, and Kawasaki Disease, Council on Cardiovascular Disease in the Young, American Heart Association. Pediatrics 2004, 114(6): 1708-1733.

57. Yang WG, Vignon-Clementel IE, Troianowski G, Reddy VM, Feinstein JA, Marsden AL: Hepatic blood flow distribution and performance in conventional and novel Ygraft Fontan geometries: A case series computational fluid dynamics study. Journal of Thoracic and Cardiovascular Surgery 2012, 143(5):1086-1097.

58. Pike NA, Vricella LA, Feinstein JA, Black MD, Reitz BA: Regression of severe pulmonary arteriovenous malformations after Fontan revision and "Hepatic factor" rerouting. Annals of Thoracic Surgery 2004, 78(2):697-699.

59. Marsden AL, Bernstein AJ, Reddy VM, Shadden SC, Spilker RL, Chan FP, Taylor CA, Feinstein JA: Evaluation of a novel Y-shaped extracardiac Fontan baffle using computational fluid dynamics. Journal of Thoracic and Cardiovascular Surgery 2009, 137(2):394-U187. 
60. Dubini G, deLeval MR, Pietrabissa R, Montevecchi FM, Fumero R: A numerical fluid mechanical study of repaired congenital heart defects. Application to the total cavopulmonary connection. Journal of Biomechanics 1996, 29(1):111-121.

61. Min J, Berman D, Shaw L, Mauri L, Koo BK, Erglis A, Leipsic J: Fractional Flow Reserved Derived From Computed Tomographic Angiography (FFRCT) for Intermediate Severity Coronary Lesions: Results from the DeFACTO Trial (Determination of Fractional Flow Reserve by Anatomic Computed TOmographic Angiography). Journal of the American College of Cardiology 2012, 60(17):B6-B6. 


\section{FIGURES:}

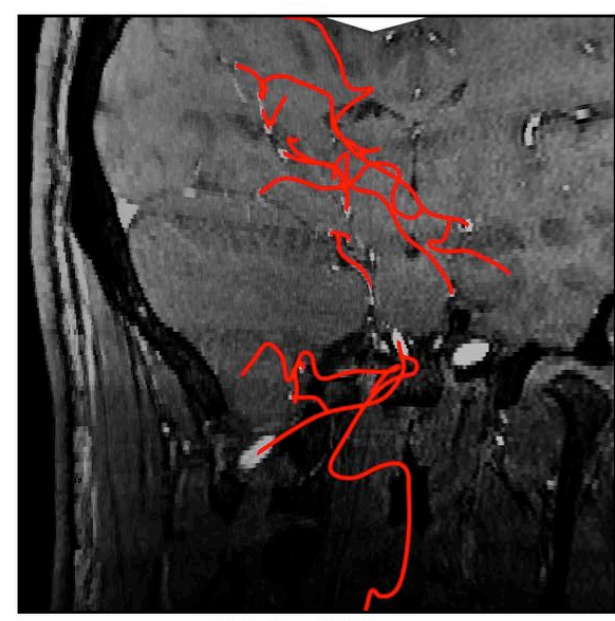

(1) Pathlines

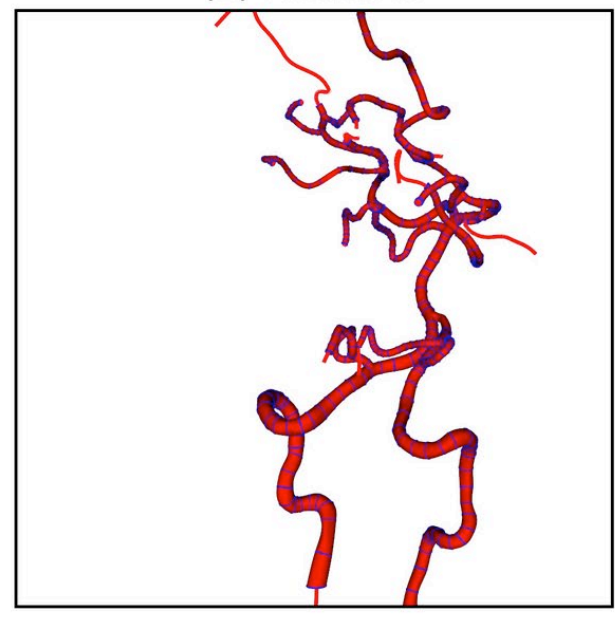

(3) Lofting

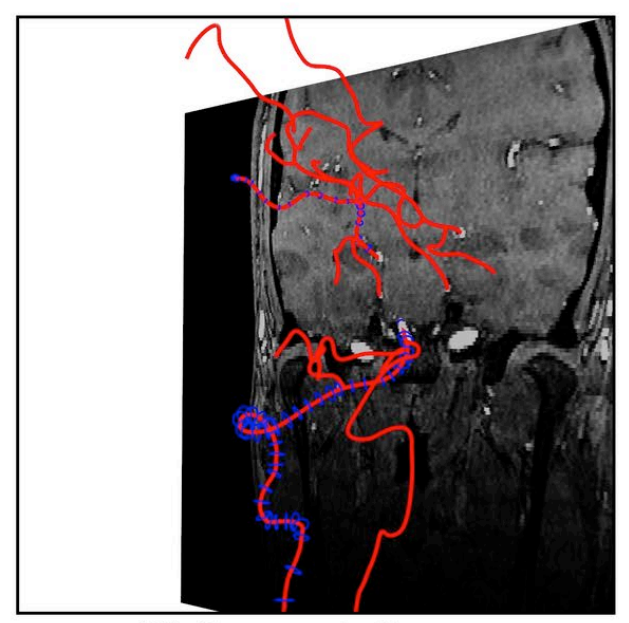

(2) Segmentations

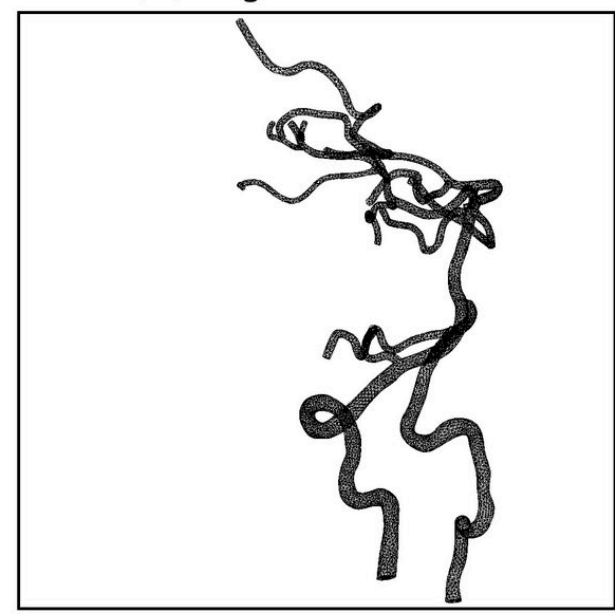

(4) Meshing

Figure 1: The steps in model construction using the Simvascular software package with 2D segmentation methods: 1) pathlines down the center of vessels of interest, 2) 2D segmentation of the vessel lumen, 3) lofting the segmentations to construct a 3D model, and 4) creation of an unstructured mesh for use in a flow solver. Example shown is construction of a patient specific model of the vertebral vasculature from MRI data. (image courtesy CVET) 


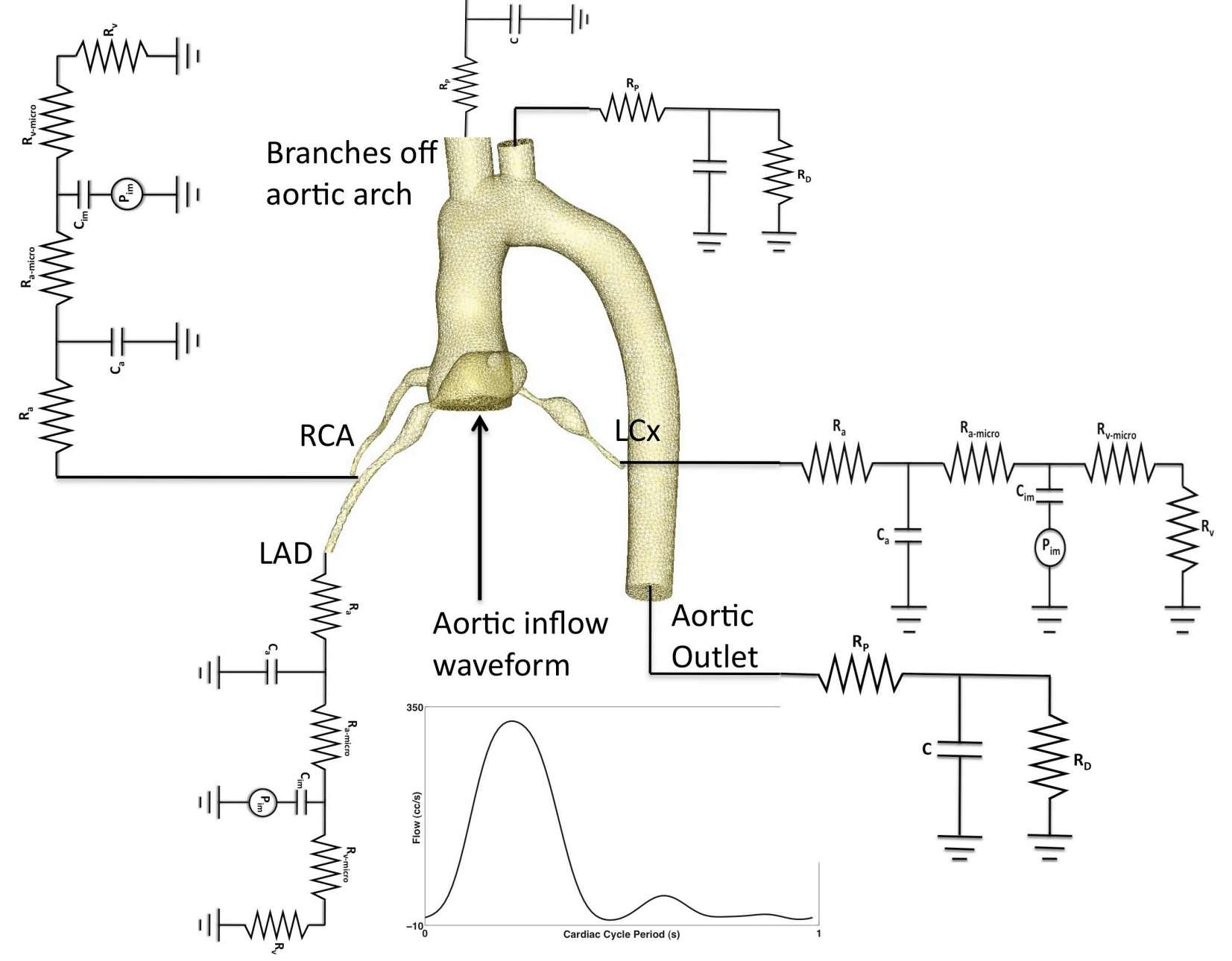

Figure 2: A typical patient specific model of the aorta and coronary arteries in a patient with Kawasaki Disease, illustrating the application of inflow and outflow boundary conditions. The model requires application of an aortic inflow condition, and lumped RCR models for the aorta and aortic branch vessels, with a specialized lumped coronary model. 
a)
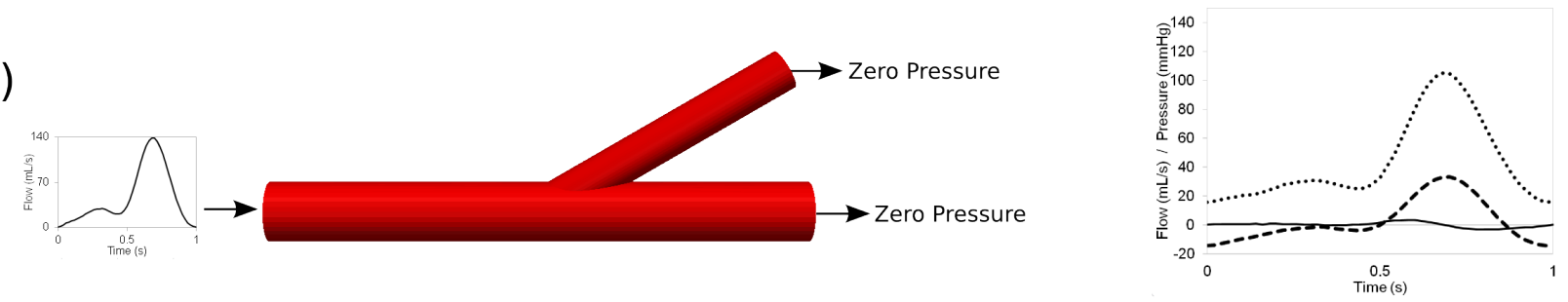

b)
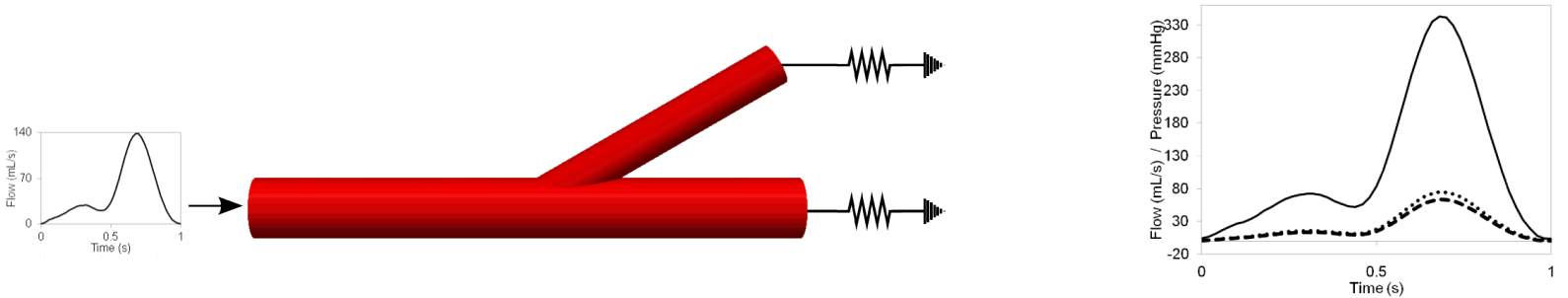

c)
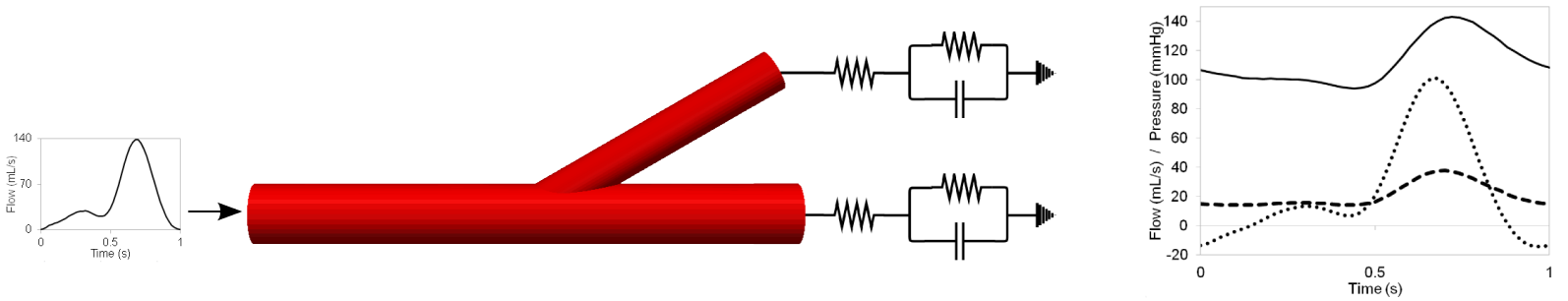

d)
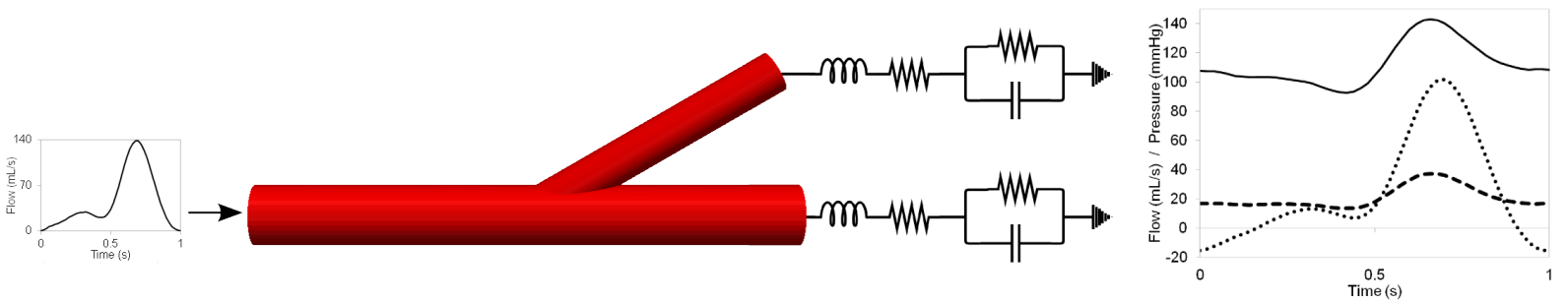

— Inlet pressure

Main outlet flow

-.- Branch outlet flow

Figure 3. Examples of Outflow BC Prescriptions and Effects on Simulation Results

a) Zero-pressure b) Resistance c) Resistance-Capacitance-Resistance d) InductanceResistance-Capacitance-Resistance 

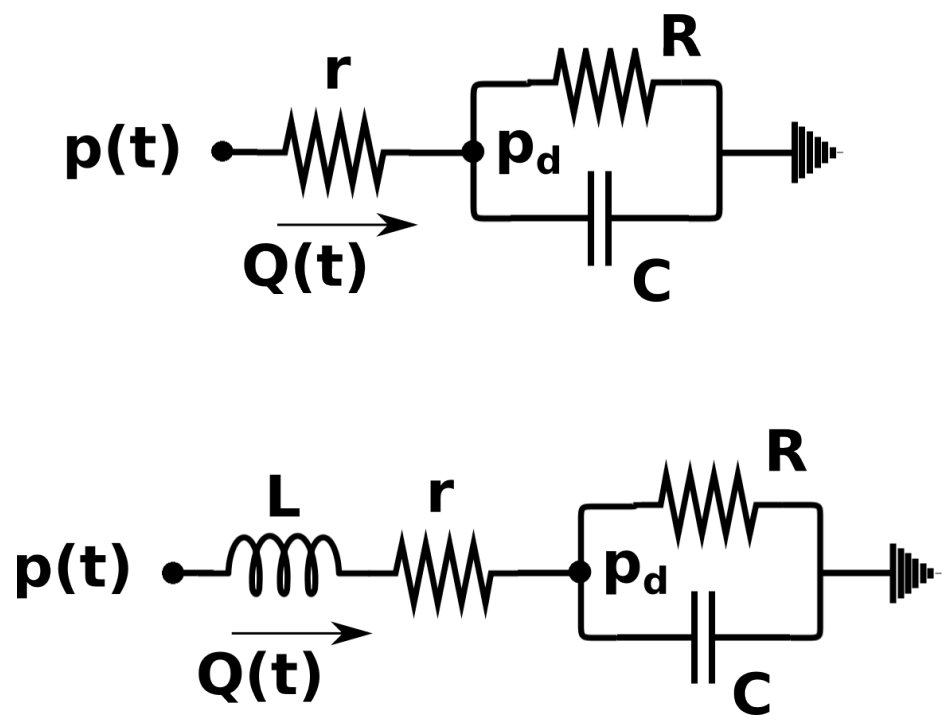

Figure 4: Lumped parameter models 


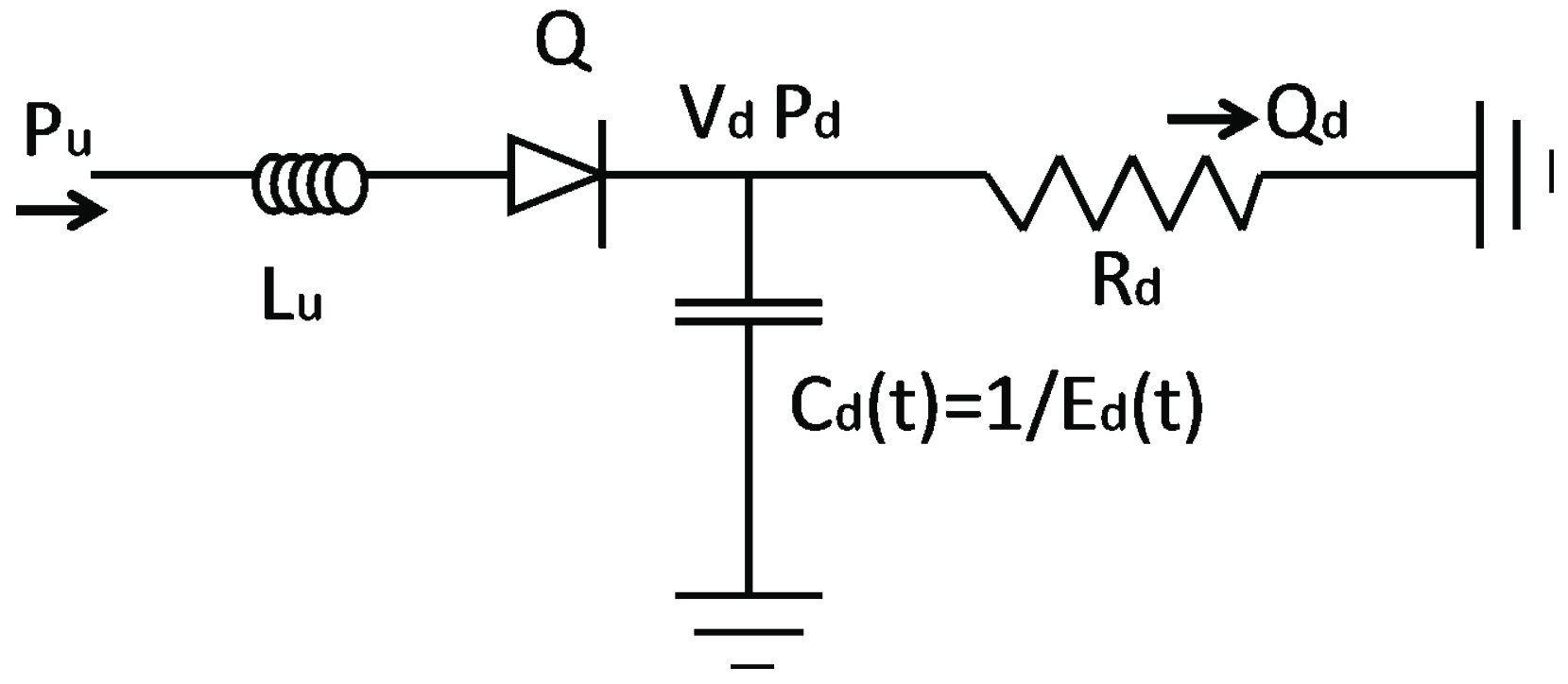

Figure 5: Sample heart model circuit 
27

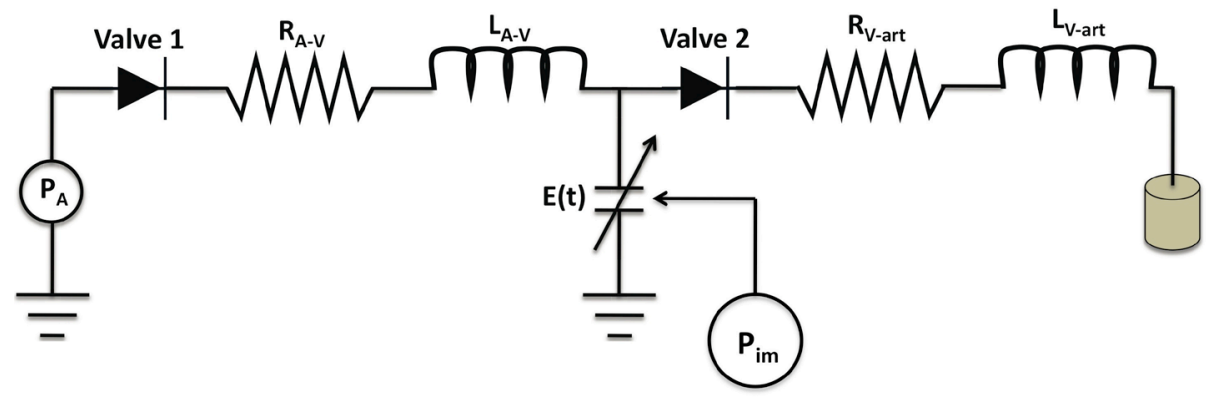

Figure 6: Sample coronary lumped model circuit 


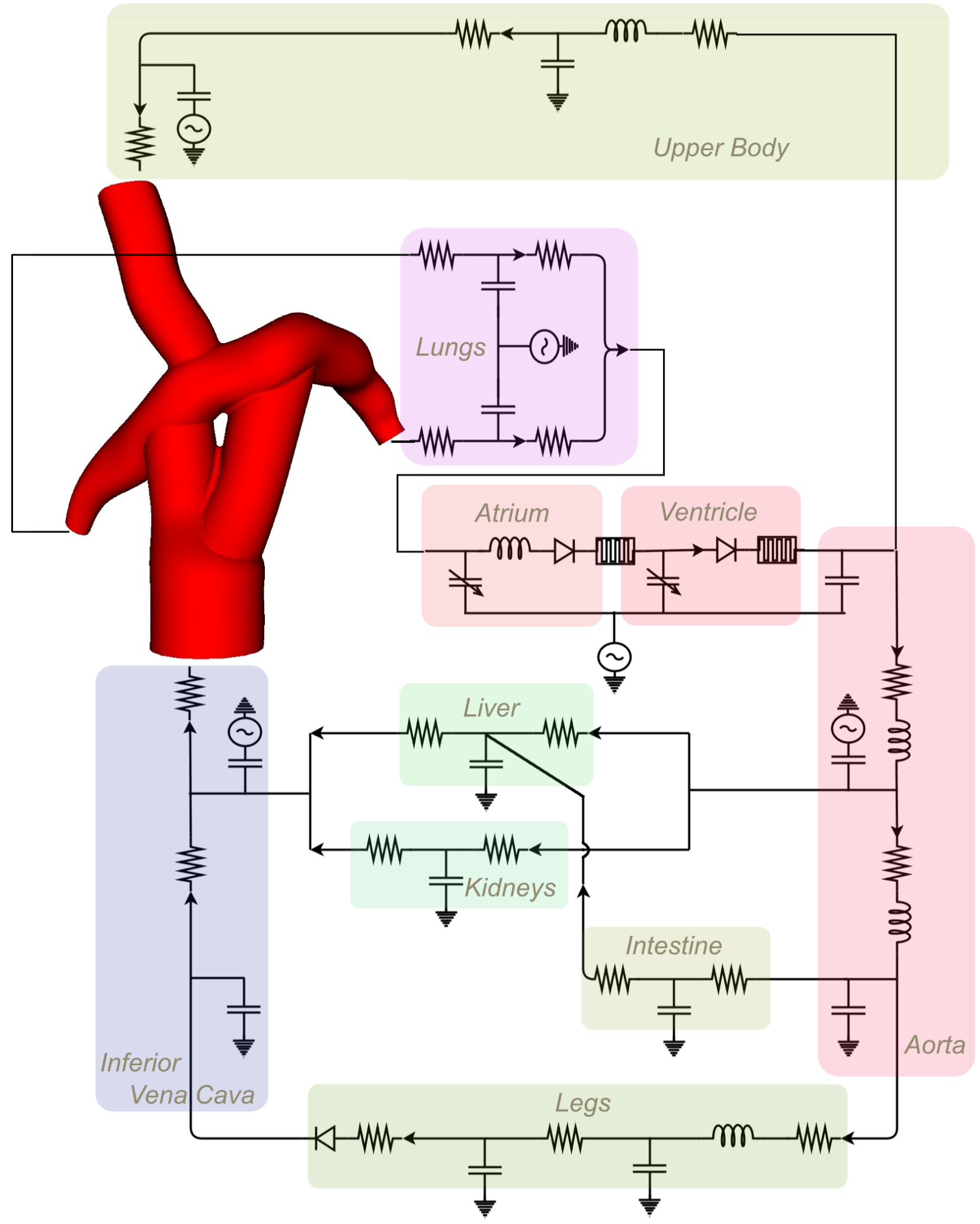

Figure 7: Sample Closed-loop 3D/0D Coupled Simulation Setup 


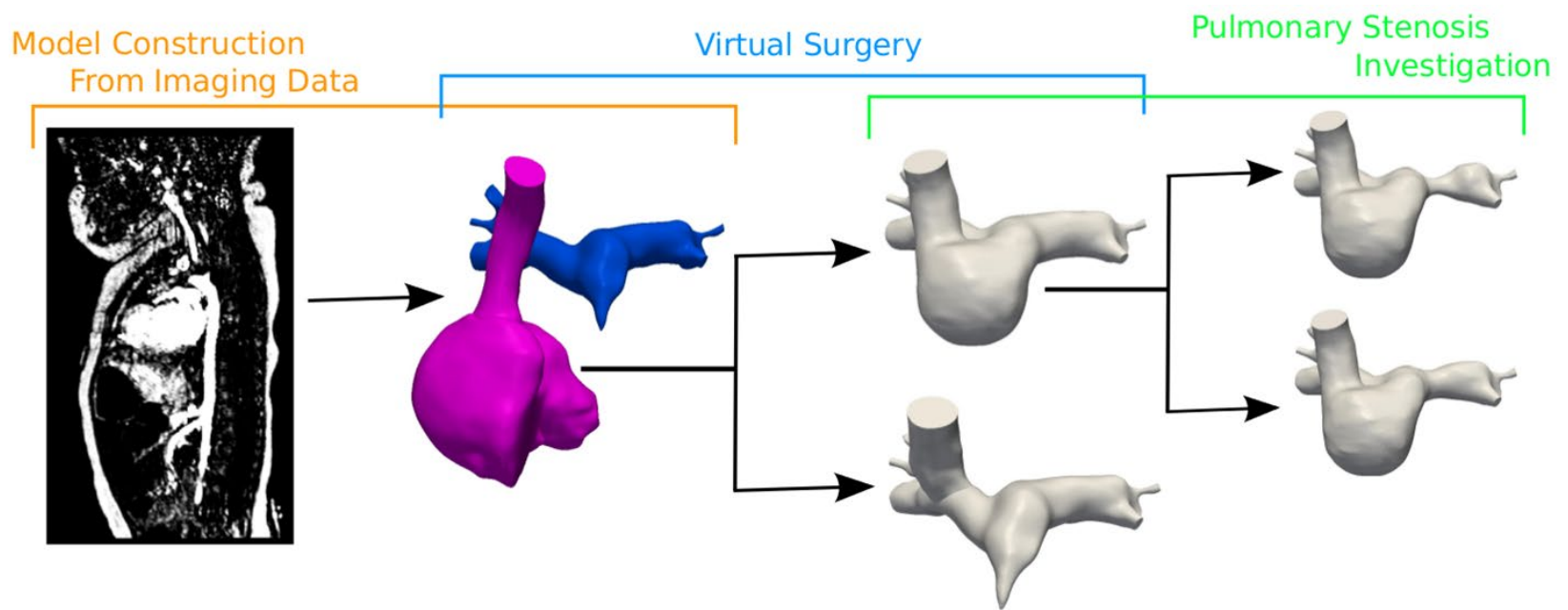

Figure 8: The process of pre-operative 3D anatomical model construction from imaging data, virtual stage 2 surgery, and virtual pulmonary stenosis

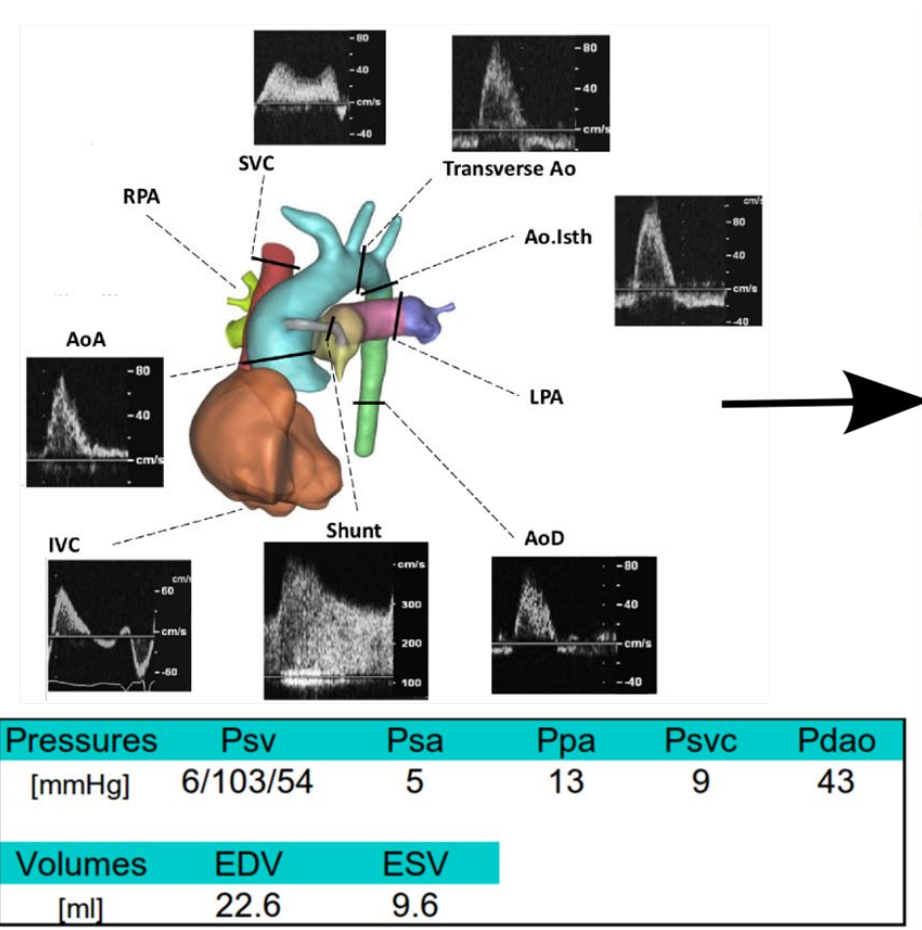

Clinical Data (MR, Ultrasound, Cath)

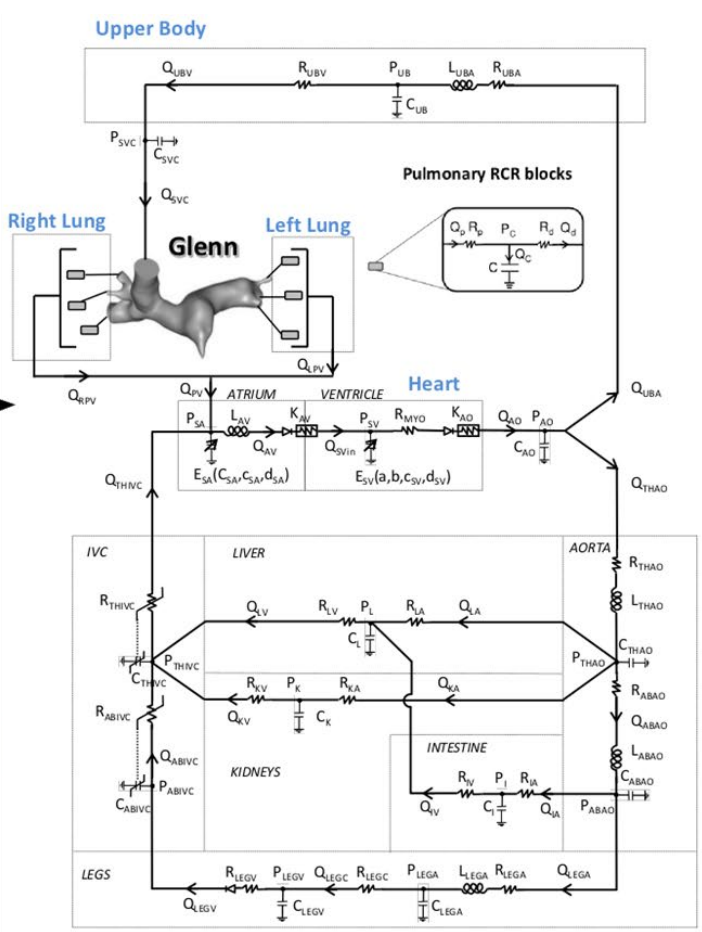

Lumped-Parameter Network Model

Figure 9: Pre-operative clinical data, and the 0D lumped-parameter network model coupled to the $3 \mathrm{D}$ anatomical model. 


\section{D Velocity Field}
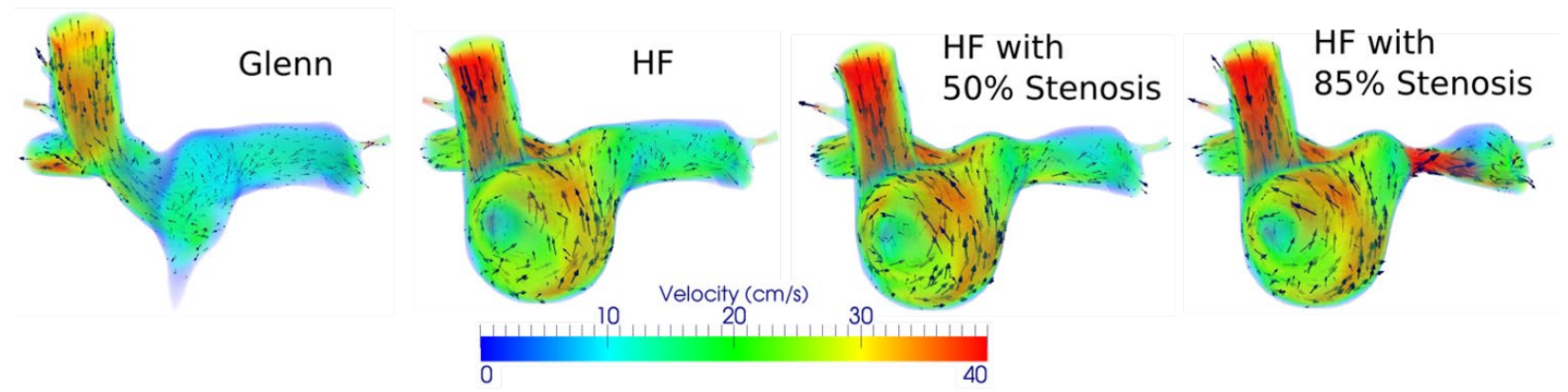

\section{D Pressure Field}
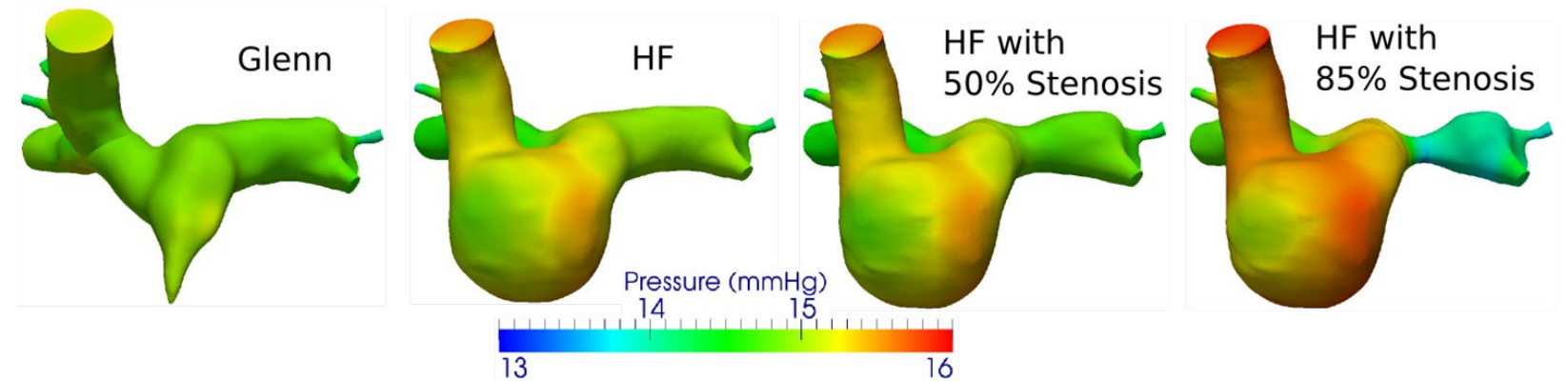

Figure 10: 3D Domain Simulation Results
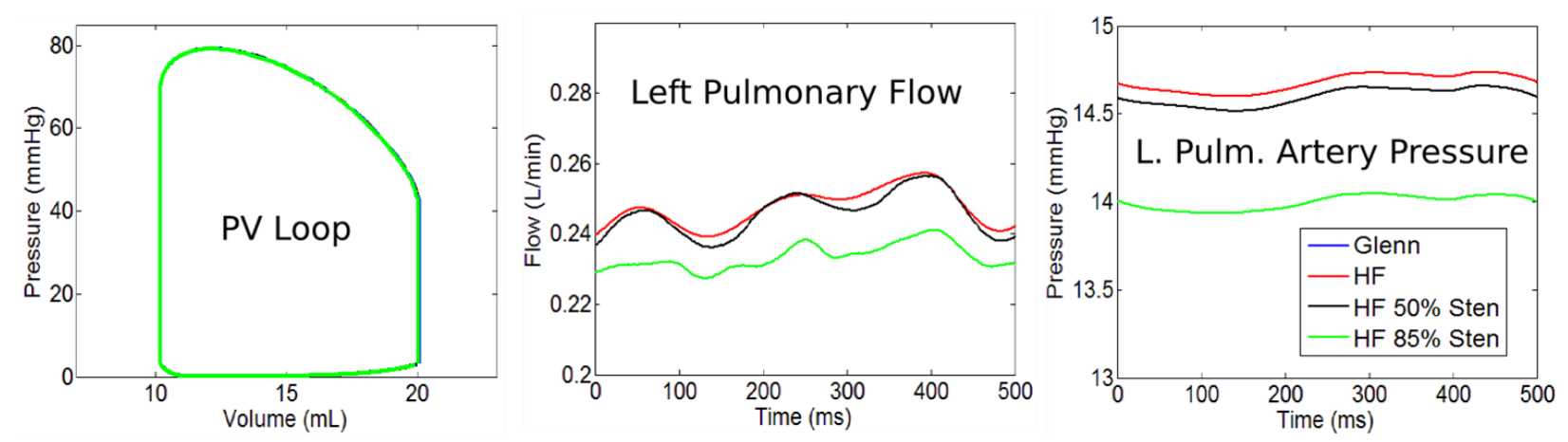

Figure 11: Time tracing of various physiological parameters
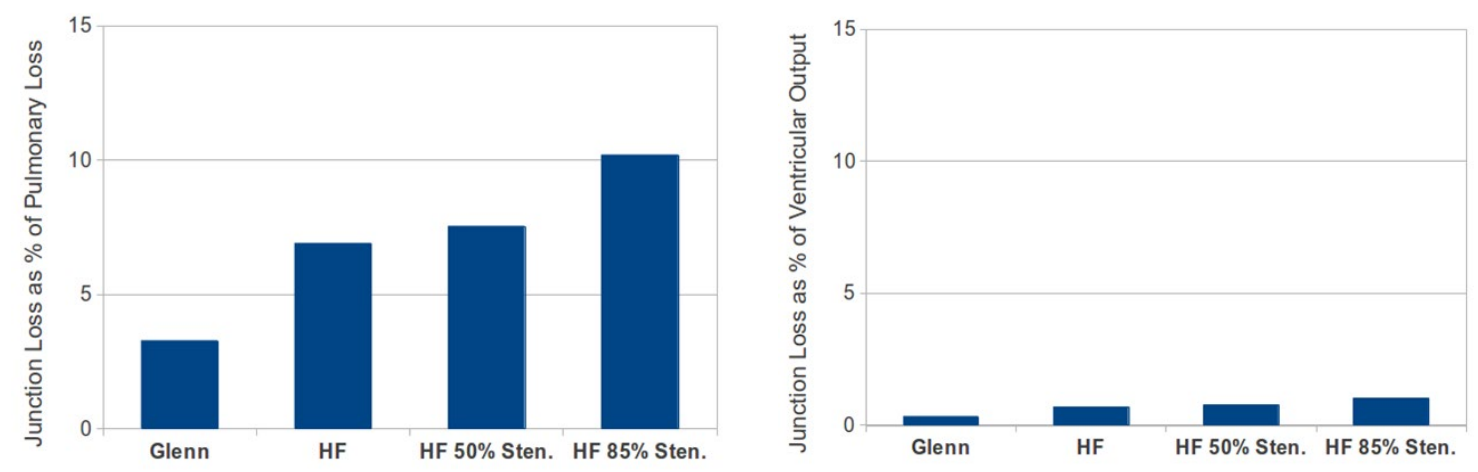

Figure 12: Power loss in the surgical junction as a fraction of other systemic powers 

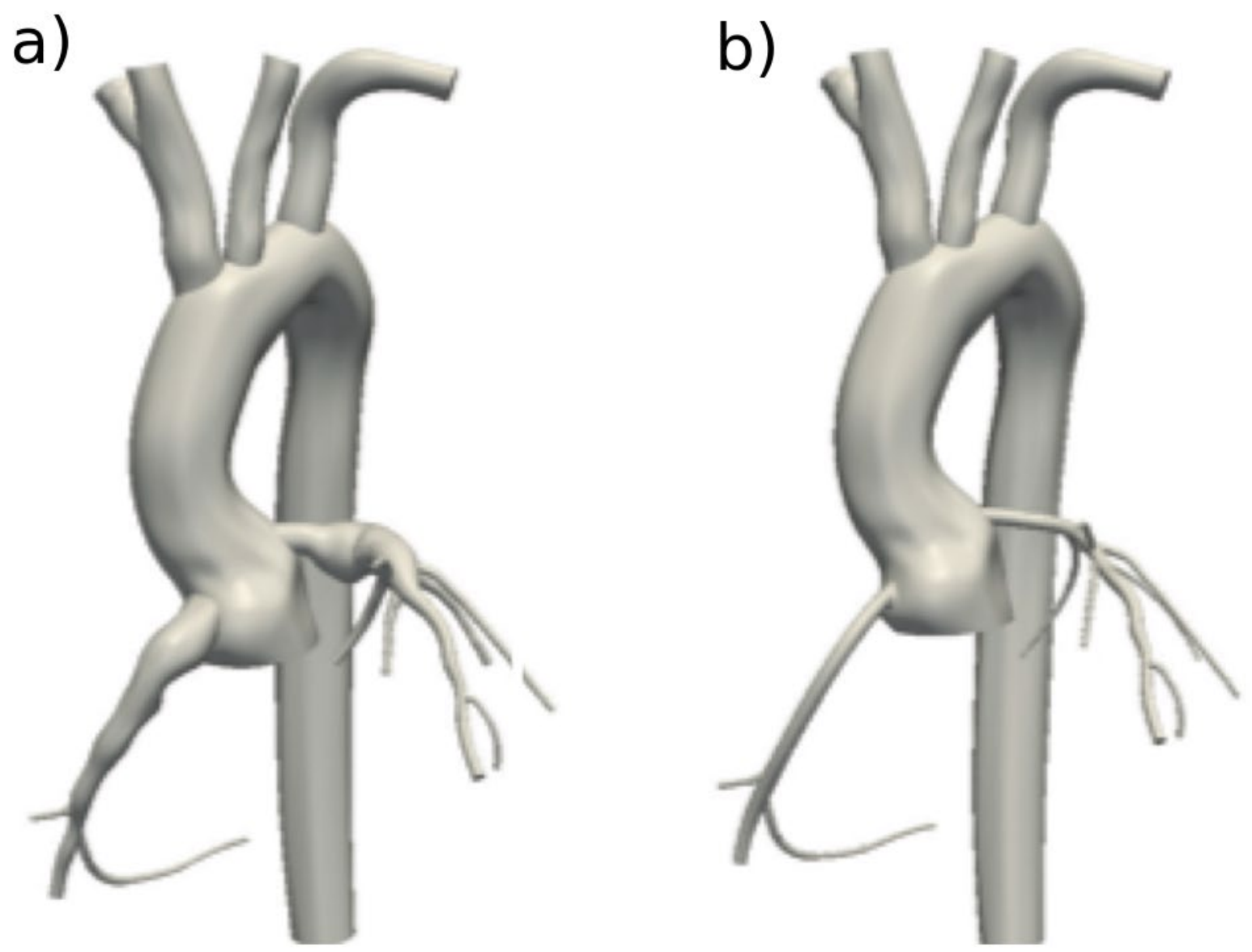

Figure 13: 3D models of aorta and coronary arteries a) with and b) without aneurysms (ref)
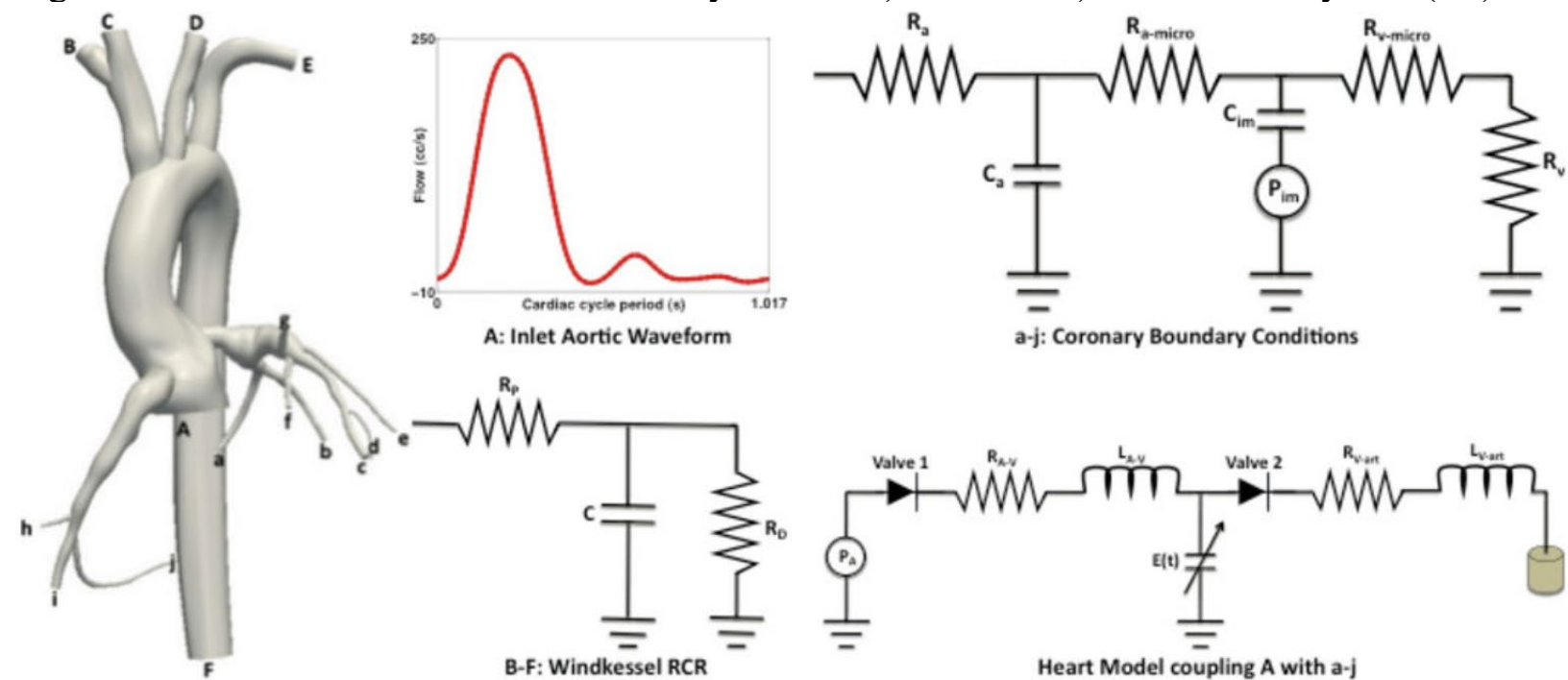

Figure 14: Multi-scale simulation setup for modeling coronary flow (ref) 

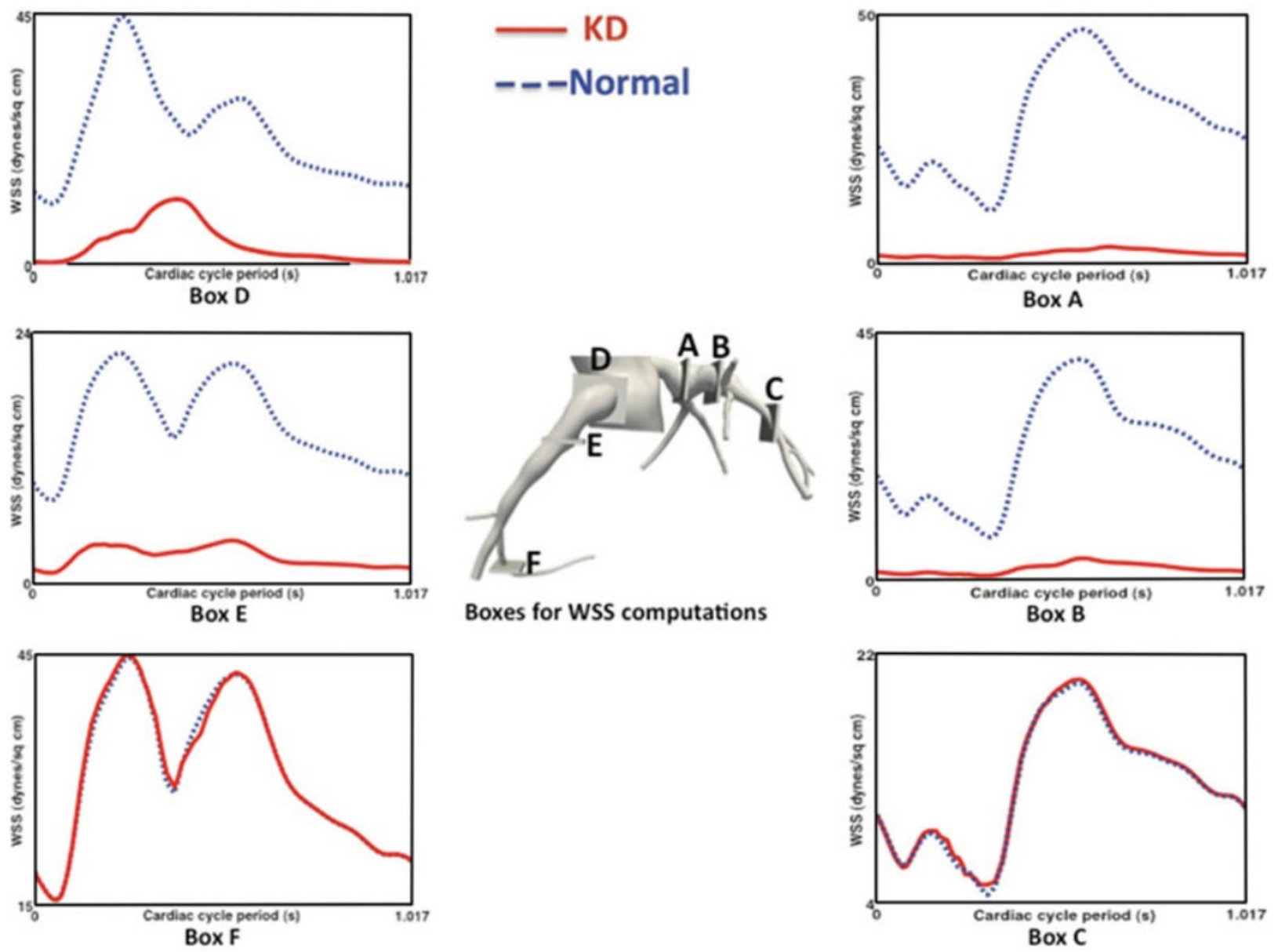

Figure 15: Comparison of spatially averaged wall shear stress (WSS) between the KD and normal cases at different locations (ref) 


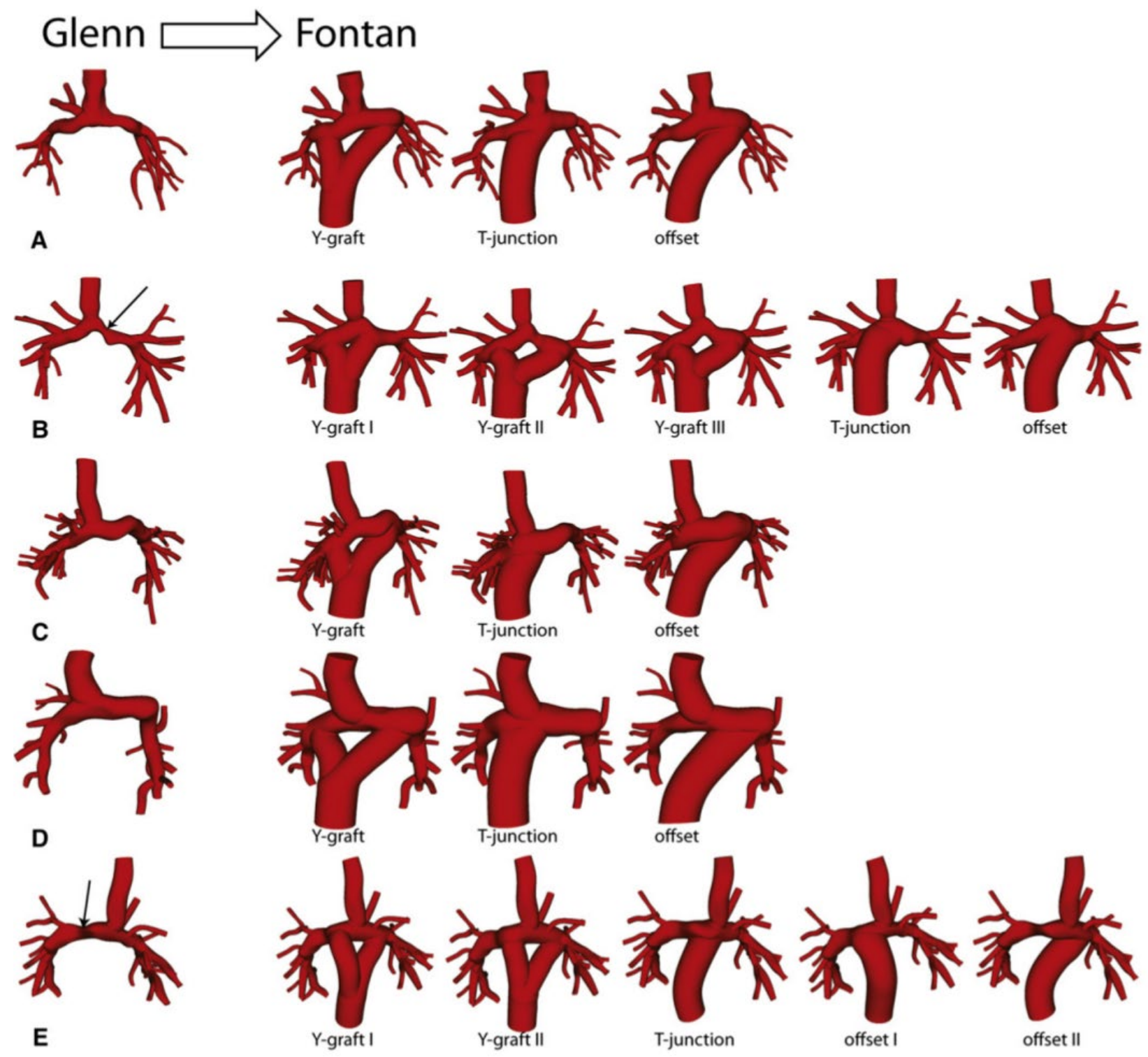

(ref) Figure 16: Pre-operative patient models and variations of Fontan geometries for 5 patients. Patients $\mathrm{B}$ and $\mathrm{E}$ have stenoses in the pre-operative anatomy denoted by arrows. 

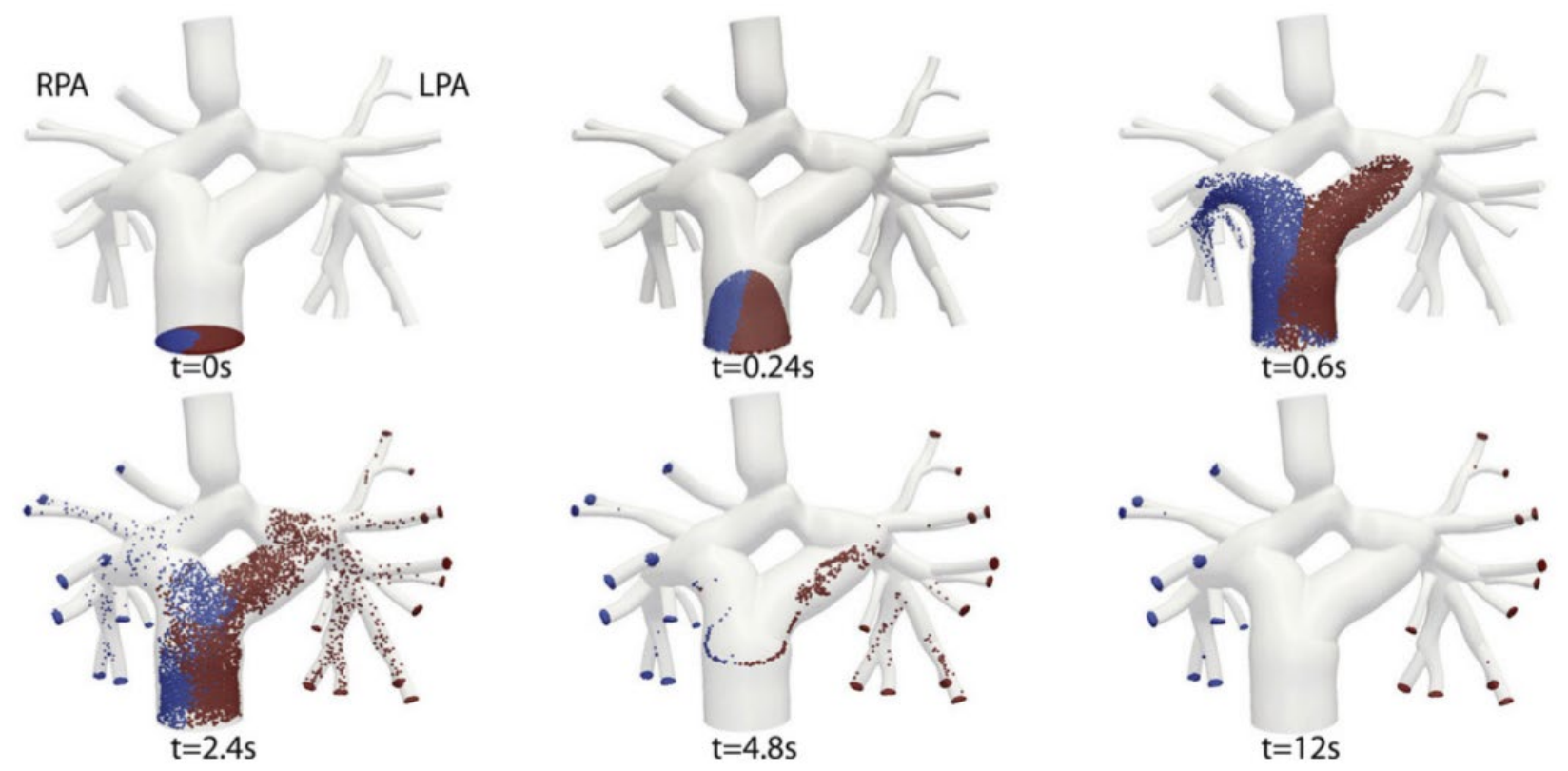

(ref) Figure 17: An example visualization of the particle tracking computation. LPA, Left pulmonary artery; RPA, right pulmonary artery. 

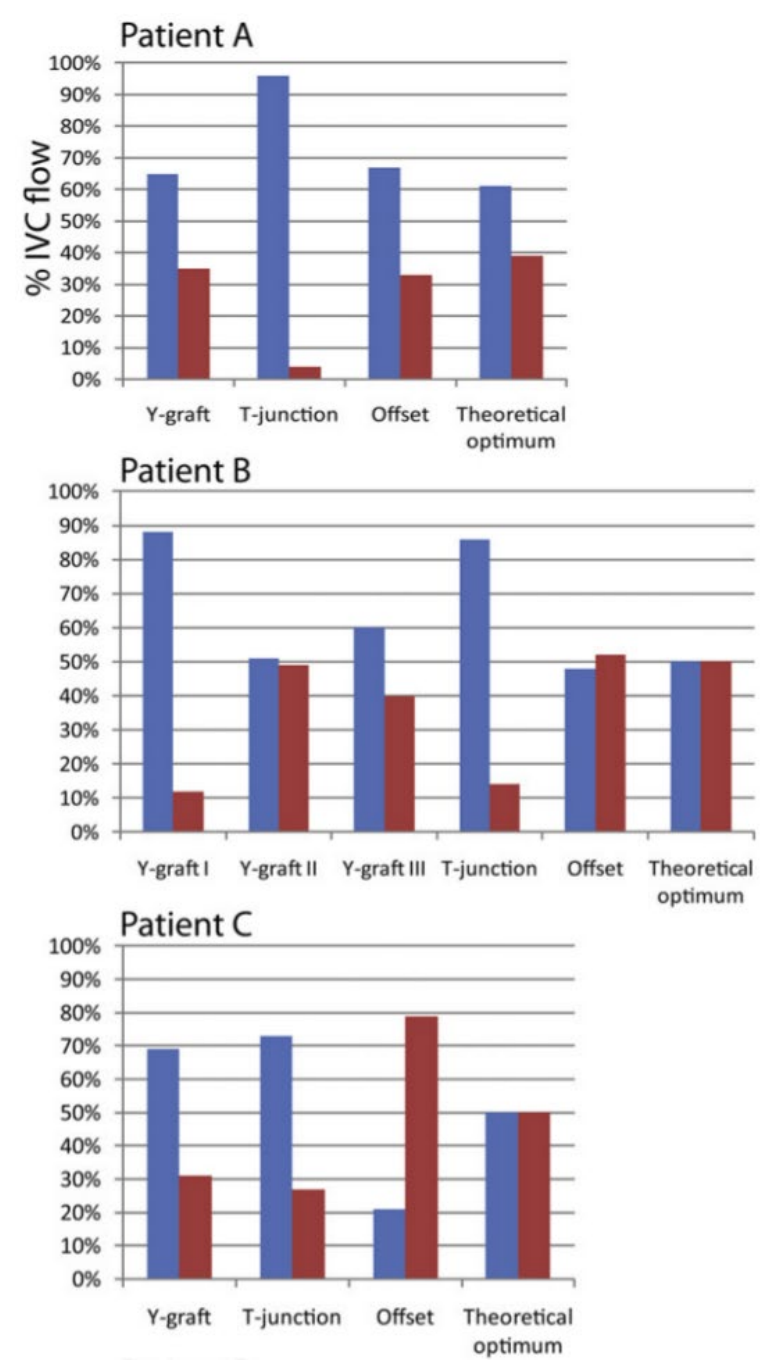
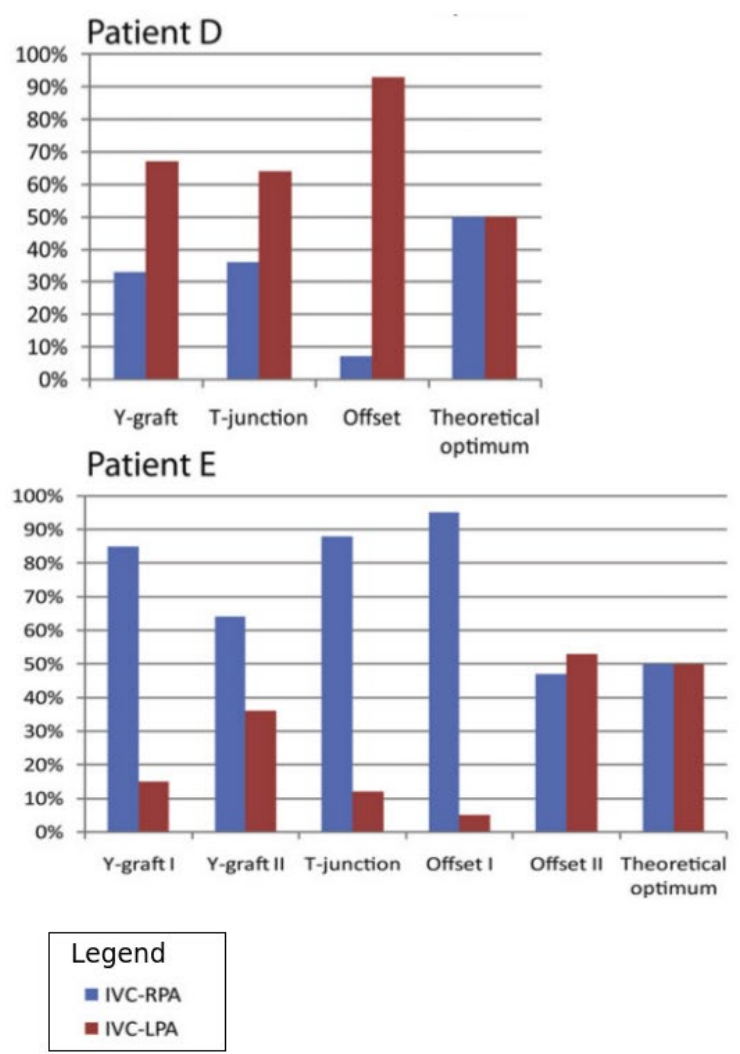

(ref) Figure 18: Hepatic flow distribution results. IVC, Inferior vena cava; RPA, right pulmonary artery; LPA, left pulmonary artery. 